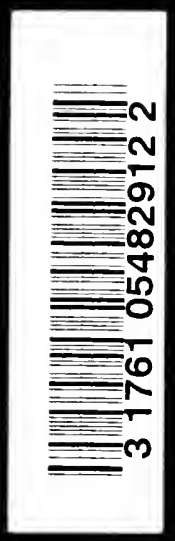



$$
+
$$
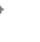

, 
Digitized by the Internet Archive in 2007 with funding from Microsoft Corporation 



\section{MODERN THEORY \\ OF \\ PHYSICAL PHENOMENA}




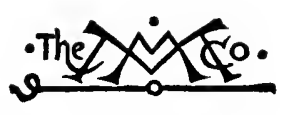




\section{MODERN THEORY}

OF

\section{PHYSICAL PHENOMENA}

RADIO-ACTIVITY, IONS, ELECTRONS

BY

AUGUSTO RIGHI

PROFESSOR OF PHYSICS IN THE UNIVERSITY OF BOLOGNA

AUTHORIZED TRANSLATION

BY

AUGUSTUS TROWBRIDGE

PROFESSOR OF MATHEMATICAL PHYSICS IN THE

UNIVERSITY OF WISCONSIN

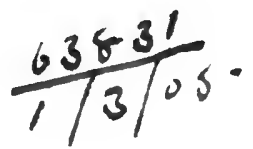

TYeñ Zigork

THE MACMILLAN COMPANY

LONDON : MACMILLAN \& CO., LTD.

I904

All rights reserved 
COPYRIGHT, 1904,

By THE MACMILLAN COMPANY.

Set up and electrotyped. Published December, rgo4. 


\section{PREFACE}

IT is with a certain trepidation that I have entertained the proposal of an English translation of an entirely unpretentious book, which is, in fact, only an extension of a chapter added to "Telegrafia senza Filo" (Telegraphy without Wires).

The fact that this book has gone through two Italian editions in a very short time certainly shows that my work has not been useless in my own country. But to issue an edition in the language of those illustrious men to whom, above all, we owe the admirable theory which forms the subject of this little book is quite a different thing.

That which has weighed with me in my decision was the thought that this English translation might at least serve to show with what great favour the development of the ideas concerning the first cause of physical 
vi PREFACE

phenomena has been received in Italy, and also to show how fully the works of those philosophers to whom this development is due are appreciated in this country.

AUGUSTO RIGHI.

BOLOGNA, ITALY,

August, I904. 


\section{TRANSLATOR'S PREFACE}

For more than twenty-five years Professor Righi has been an indefatigable investigator and constant contributor to electrical science, and has attained a foremost rank among Italian scientists. While he is not one of the relatively small group of investigators to whom we owe the extremely important theory outlined in this book, he is, nevertheless, admirably qualified to discuss and explain the theory, both by reason of his deep insight into electrical phenomena and because of his ability to explain intricate physical processes without the aid of mathematical formulæ.

As Professor Righi states in his preface to the first edition, the Italian original was written more with the object of interesting the greatest possible number of readers in this new and important branch of physics 
than as a book of reference for physicists. With this end in view the subject-matter was presented in an elementary form, and the book met with a very marked success.

I have made this translation believing that there are at least as many English as Italian readers to whom an elementary treatment of the electron theory as it stands at present will be acceptable.

Professor Righi has read the proofs of the translation, thus insuring its accuracy, and has kindly provided me with a special preface in English.

AUGUSTUS TROWBRIDGE.

MADISON, WISCONSIN,

September, IgO4. 


\section{CONTENTS}

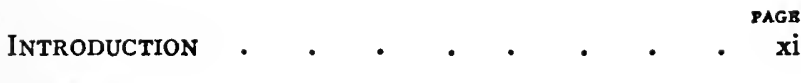

CHAPTER

I. Electrolytic Ions and Electrons • - I

II. The Electrons and the Phenomena of LIGHT • • • • • • • • II

III. Nature of the Cathode Rays • $\quad$ - 28 IV. The Ions in Gases and in Solids • . 40

V. RADIO-ACTIVITY

VI. Mass, Velocity, and Electric Charge of THE IONS AND OF THE Electrons • . 108 VII. The Electrons and the Constitution of MATTER • . • . BIBLIOGRAPHY • • • • • • • • 



\section{INTRODUCTION}

A NEW and interesting branch of science has been formed, partly as a result of the numerous recent experimental researches on the electric discharge, and partly as a consequence of the discovery of radio-activity and new phenomena in magneto optics. At the same time these researches have caused a theory to spring up which harmonizes all the facts, and which has profoundly modified the dominant ideas concerning the immediate cause of electrical phenomena, and of physical phenomena in general.

When the old hypothesis of the electric fluid was abandoned, chiefly because of the disinclination to admit "action at a distance," it seemed for a time as though Faraday's ideas, formulated later by Maxwell, must lead to a new concept regarding the cause of electrical phenomena, since, according to Faraday, the ether, and not the so- 
called electrified bodies, is the seat of the phenomena. But the impossibility of finding a satisfactory mechanical representation of the supposed elastic deformations of the ether, to which were attributed in Maxwell's theory the apparent forces at a distance, and the necessity of admitting at any rate the existence of an entity distinct from the ether and from matter, made it soon apparent that, even under the new order of ideas, the conception of the nature of electricity still remained cbscure.

At present a new evolution is being accomplished, since, without lnowing anything more about the first cause, an atomic structure is attributed to electricity. This new conception, suggested by the studies mentioned farther on, already shows promise of becoming as fruitful as the analogous one which has long been admitted regarding the constitution of matter, inasmuch as it permits us to place in reciprocal relation, often even quantitative, phenomena which seem utterly different and independent of each other. 
What the electrons or electric atoms really are remains a mystery; but in spite of this the new theory may perhaps acquire not a little importance in the future even from the philosophic point of view, since it points out a new mode of considering the structure of ponderable matter and tends to bring back to a single origin all the phenomena of the physical world.

It is indeed true that, with the modern positivist and utilitarian tendencies, many people do not appreciate this advantage, and prefer to consider a theory principally as a convenient means to arrange and coördinate facts, or as a guide for the investigation of new phenomena. But if hitherto men have confided too much in the power of human ingenuity and have too easily believed themselves to be on the point of discovering the ultimate cause of things, we perhaps fall into the contrary excess.

In this book I shall set forth the principal facts which have led to the electron theory, and I shall endeavour to make this theory clear at least along its general lines. 


\section{THE MODERN THEORY OF PHYSICAL PHENOMENA}

\section{CHAPTER I}

ELECTROLYTIC IONS AND ELECTRONS ${ }^{1}$

THE hypothesis of electrolytic dissociation is generally admitted in order to explain electrolysis in accord with the well-known laws of Faraday which this phenomenon obeys. Each molecule of an electrolyte may break up into two ions; that is, into two atoms or atomic groups having equal charges of opposite sign. Thus when a solid, such as chloride of sodium, or common salt, is dissolved in water, some of its molecules undergo dissociation; that is, these molecules cease to exist as such, and their ions become separated and free. Owing to the

1 The numbers in parentheses inserted in the text refer to the bibliography at the end of the book. 
invisible molecular and atomic motions, whose energy constitutes the heat contained in a body, these ions wander through the liquid, without, however, any given direction of motion predominating. In the resulting mutual collisions it happens at one instant that a molecule breaks up into ions, at another instant that individual ions recombine into molecules. There are, so to speak, incessant unions and separations, in spite of which the number of dissociated molecules remains sensibly independent of the time.

When two electrodes connected with the poles of a battery are immersed in the solution, the ions of the two kinds - for example, the positive ions of sodium and the negative ions of chlorine - no longer wander at haphazard in any direction; but, obeying the electric force, the first approach the negative electrode, or cathode, the second the positive electrode, or anode. On arriving at the electrodes, the ions give up their charges and become neutral atoms, which remain free, at least if no special chemical action takes place between these atoms and 
the surrounding bodies (this, by the way, is precisely what would happen in the case of the sodium). The electric current in the liquid consists in this transport of electricity brought about by the ions.

Electrolysis obeys the two laws which were enunciated by Faraday. The first of these laws, which asserts the proportionality existing between the quantity of electricity traversing the liquid and the quantity of matter deposited on the electrodes, is embodied in the statement that all the ions existing in the liquid possess charges equal in absolute value. Thus, in the case of chloride of sodium, the ions of the metal have positive charges all equal among themselves, and equal, but of opposite sign, to the charge of any one of the ions of chlorine.

In order to satisfy Faraday's second law, according to which a proportionality exists between the relative chemical equivalents of different electrolytes and the amount of each decomposed when the same quantity of electricity is transmitted through them all, as would be the case when all were placed in 


\section{4 ELECTROLYTIC IONS AND ELECTRONS}

series in a single circuit, it is necessary to admit that all univalent atoms possess a charge equal in absolute value to that of the sodium or of the chlorine ion: that all atoms which possess twice this charge behave as bivalent, etc. The following example will make this point clear. If a current is passed through a solution of the chloride of copper the molecules of which contain two atoms of copper (univalent) and two of chlorine, and also through a solution of the other chloride, the molecules of which contain one atom of copper (bivalent) and two of chlorine, there will collect on the cathode of the first solution a quantity of copper double that on the second cathode, although, naturally, the quantity of positive electricity transported through the two liquids will have been the same.

In $188 \mathrm{I}$ the illustrious Helmholtz pointed out that the laws of electrolysis suggest the idea that the electric charge pertaining to any valency of an ion may be a fixed quantity having a separate existence; and, since a material atom is a fixed and determinate portion of a certain kind of matter and is 
considered indivisible, it is thus natural to consider this electric charge as fixed and indivisible, all the more because a quantity of electricity smaller than this is never encountered. The charge of the ion (univalent) may therefore be called an atom of electricity, or better, as Mr. Stoney has proposed, an electron (electric ion).

In fact, as early as 1871 , considerably in advance of Helmholtz, Weber conceived the idea of the atomic structure of electricity. This famous physicist and mathematician proposed the well-known theory, according to which electric phenomena are due to particles or atoms of positive and negative electricity acting on each other at a distance, with forces depending, not only upon the distance itself, but also on the velocity of the particles and on their accelerations; that is, on the manner in which these velocities vary. Naturally this theory in which action at a distance was still admitted has nothing in common with that now in favour except the fundamental concept of the electric atom; and although Weber, searching in his theory 
for the cause of the forces which govern the atomic structure of bodies; advanced the hypothesis "that to every ponderable atom there is united an electric atom" ( $\mathrm{I}$ ), the relation now admitted between ions and electrons seems to have been better perceived by Helmholtz.

We do not believe that the atomic hypothesis concerning the nature of electricity imposes on us the necessity of considering it as matter, since we are still free to suppose than an electron may be simply a special localized condition of the universal ether. We may, on the contrary, from now on add that instead of considering electricity as matter, we are led to the exactly opposite hypothesis that the atoms of various bodies are systems of electrons.

When the ions arrive on the electrodes and become neutral atoms, the electrons enter into the circuit to constitute the electric current. Now it seems natural to suppose that these electrons, instead of merging, so to speak, into a homogeneous whole (the old electric fluid), preserve their individual- 
ity; this is all the more natural because, if they are to pass from one atom to another, it is most probable that they must exist momentarily isolated; thus the electric current in conductors would be nothing else than a motion of free electrons across interatomic space. It remains undetermined whether the current consists in the motion of positive electrons in one direction and negative in the opposite direction, or in the motion in a given direction of one of the two kinds of electrons, say the negative; but preference is given to the latter opinion, because, while there is reason to hold that the negative electrons may exist in a free state, this is not true of the positive electrons. Only the former, as it appears, suffer displacement, separate themselves from ponderable matter or unite with one another, and vibrate in light sources, as we shall soon see. Therefore, while a negative ion in being deposited on the anode gives up its electron, a positive ion arriving at the cathode does not give up the positive electron, but takes away a negative electron from the cathode itself. 
Here, then, is the old theory of the electric fluid in a certain sense called back to life, but profoundly modified. It is no longer a question of a continuous fluid, but of special atoms (the electrons), which, however, as has already been observed, are not necessarily to be considered as material in the ordinary sense of the word.

Besides, and this is of more importance, we do not attribute to the atoms of electricity that mysterious faculty of acting at a distance, with which the old fluid was supposed to be endowed, but instead we suppose that the reciprocal forces between the electrons have their origin in the special elastic deformations of the ether, identical with those called for in Maxwell's theory to take account of the electric forces between conductors.

To explain the phenomena of electrolysis it is sufficient to admit, as is always done, the hypothesis of electrolytic dissociation; but this hypothesis is not well adapted to explain the propagation of electricity in gases and certain other phenomena. How- 
ever, with the admission of electric dissociation, - that is, the separation of the negative electrons from the neutral atoms, - we assign a reason both for electrolysis and for these other phenomena as well.

In order that a negative electron may separate itself from a neutral atom, energy must be expended to overcome the attraction by which the electron is held to the positive ion, which is what remains of the atom when the negative electron is taken from it, precisely as it is necessary to furnish heat energy to separate the molecules of a liquid from one another in evaporation, or as it is necessary to do mechanical work in lifting a weight from the earth.

The energy necessary to ionize or dissociate an atom naturally varies according to its chemical nature. Experiment indicates that this energy is a minimum for the so-called electropositive bodies, such as the metals, and gradually becomes greater as we proceed toward the more electronegative bodies, which, moreover, may even take on new negative electrons. This energy depends 
also upon the nature and on the condition of the atoms surrounding the one which is about to break up into an electron and a positive ion; it is extremely small for bodies in aqueous solution.

This being so, electrolytic dissociation, or the separation of a molecule into two ions, for example, sodium chloride into a positive ion of sodium and a negative ion of chlorine, - should be considered to be a consequence of the dissociation of the metallic atom. This atom breaks up into a positive ion of sodium and into a negative electron, which is seized by the chlorine atom, transforming the latter into a negative ion. Once we adopt this mode of considering electrolytic dissociation, it, with all its very important consequences, enters into the more general electron theory. 


\section{CHAPTER II}

THE ELECTRONS AND THE PHENOMENA OF LIGHT

WhILE the hypothesis of the electrons springs in such a natural manner from electrolytic phenomena, it is in an entirely different field of physics, namely, that of optics, that it finds an unexpected and brilliant confirmation.

It is a fact, now recognized by all, that light is a vibratory phenomenon, and may no longer be considered to be due to the emission of discrete corpuscles by luminous bodies, as Newton supposed. In support of this many beautiful and classic experiments exist, with which the names of Young, of Fresnel, and of Foucault are connected. And when we speak of light, we necessarily include radiant heat, because since the celebrated researches of Melloni there can exist no doubt concerning the identity of the 
nature of these phenomena, which appear to be so different.

But the undulatory theory demands a medium capable of propagating the waves; from this arises the necessity of admitting the existence of the ether; that is, of a substance distributed everywhere throughout interplanetary and interstellar space and throughout interatomic space as well. The hypothesis of the ether forces itself on us in an irresistible manner, and almost seems to acquire the character of reality and certitude, when we consider the perfection with which the undulatory hypothesis takes account of all optical phenomena even quantitatively and in the minutest detail.

Following the example of Fresnel, light vibrations were considered for a long while to be true mechanical vibrations of the ethereal and material particles, but later it was recognized, especially in consequence of the work of Maxwell, that light waves could be considered as electromagnetic waves; thus two distinct classes of physical phenomena were united. The electromagnetic 
theory of light has received in recent years a material support from the well-known experiments of Hertz, and later from those of other physicists; and at present there is perhaps no one who will refuse to admit that the phenomena of light are in reality electromagnetic phenomena, and that light waves differ from those which Hertz has shown how to produce, only in so far as numerical values are concerned.

However, the electromagnetic theory of light, derived as it is from the properties of the electromagnetic field, does not serve to explain those phenomena to take account of which it was necessary, under the old mechanical theory, to resort to an action of ponderable matter on the ether. To complete the theory accepted at present, it was therefore necessary to take account, in some manner, of the material atoms; and the Dutch physicist, Lorentz, had the fortunate idea of considering the electric charges of the atoms together with the atoms themselves. If merely the negative or merely the positive charges take part in the light 
vibrations, and if both the electric and magnetic forces generated by their motion be taken into account, one arrives at an electromagnetic theory of light capable of explaining even those phenomena which eluded the theory based simply on the formula of Maxwell or of Hertz.

Let us here consider a most interesting phenomenon discovered by Zeeman, a former pupil of Lorentz, because it is one of those by which the relative independence of the negative electrons and their characteristic freedom of motion is most clearly demonstrated.

It is a well-known fact that a luminous gas emits radiations of definite periods of vibration, and not those corresponding to a continuous series of intermediate periods. As a result the spectrum of the light emitted by the gas reduces to a limited number of narrow lines, which are the images of the slit through which the light is passed in order to analyze it with the prism. For example, the spectrum of the light emitted by sodium in the gaseous state consists of two 
yellow lines very near each other, which with low-power spectroscopes appear blended into a single line. Now Zeeman showed that if a gas is placed in an intense magnetic field, say between the poles of a powerful electromagnet, each simple line of its spectrum is in general broken up into a group of new lines.

There are two important cases to be considered: First, that in which the luminous ray which we are considering is parallel to the lines of magnetic force; second, that in which the ray is perpendicular to the lines of force. The general case is naturally a trifle complicated, and for its treatment I refer the reader to original articles on this subject (2).

Let us suppose that we have a luminous gas between the two opposite magnetic poles; for example, vapour of cadmium obtained by passing electric sparks between two wires of that metal. If we examine the light which is propagated in the direction of the lines of force (Case I), that is, from one pole toward the other, we easily ascertain that, while the green line of the spectrum of cadmium ap- 
pears sharp and simple as in $\vec{A}$ (Fig. I) before the magnetic field is set up, the instant the field is created the line $A$ vanishes, and instead of it there appear two new lines, $B$ and $C$, one on either side of the position $A^{\prime}$,

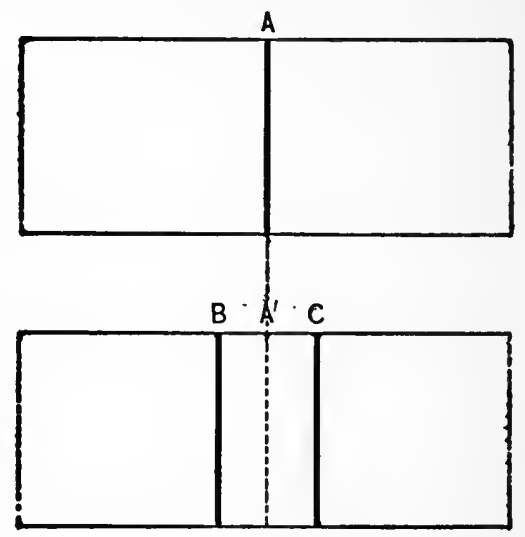

FIG. I.

which the single line at first occupied, and at equal distances from it.

If we examine with the spectroscope a ray of light in the equatorial direction (Case II), that is, perpendicular to the direction of the magnetic field, the single line, $A$ (Fig. 2), is replaced by three lines, $C, A^{\prime}, B$, of which the one in the middle, equidistant from 
the other two, occupies the position of the original line.

In the case of other spectrum lines, either the phenomena are identical with those which are exhibited by the green cadmium

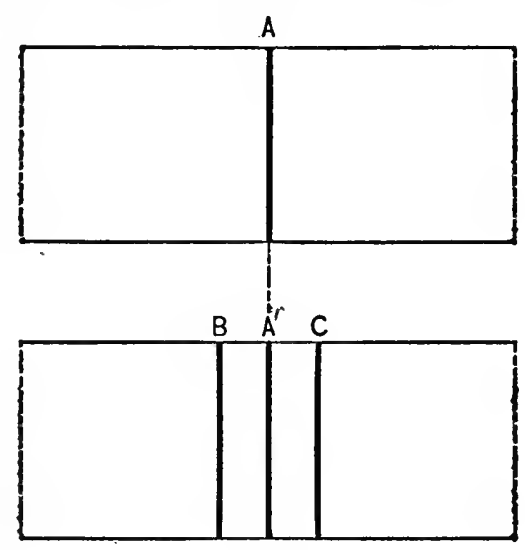

FIG. 2.

line, or slightly more complicated effects are obtained. Thus, for example, of the two sodium lines, the one usually called $D_{1}$ is transformed in the second case into four lines, $A, B, C, D$ (Fig. 3), while the line $D_{2}$ changes into a group of six lines (Fig. 4), $A, B, C, D, E, F$.

Complete explanation of these phenomena, 
at any rate in the least complicated cases, is furnished by Lorentz's theory; but for our purpose it will be sufficient to treat the single case, to which Figure I refers, of light emitted by the vapour of cadmium in the direction of the lines of force.

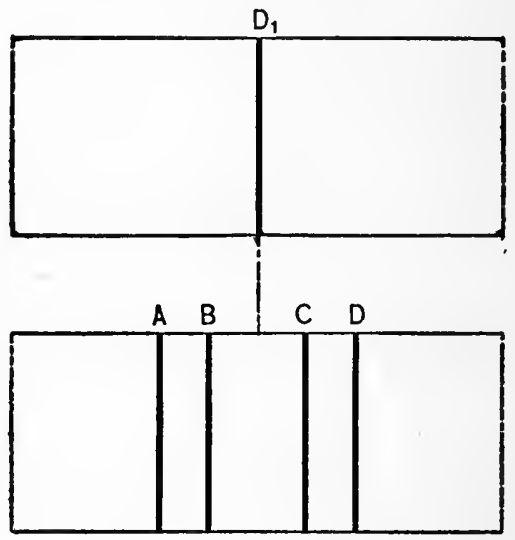

FIG. 3.

Let us consider an electrified particle which, attracted toward a position of equilibrium, $O$ (Fig. 5), vibrates about this point with circular motion, describing a circumference of radius $O A$. The vibrating electrified particle generates light waves. Suppose we study the light which is propagated in 
the direction perpendicular to the plane of the circumference. If a magnetic force acts

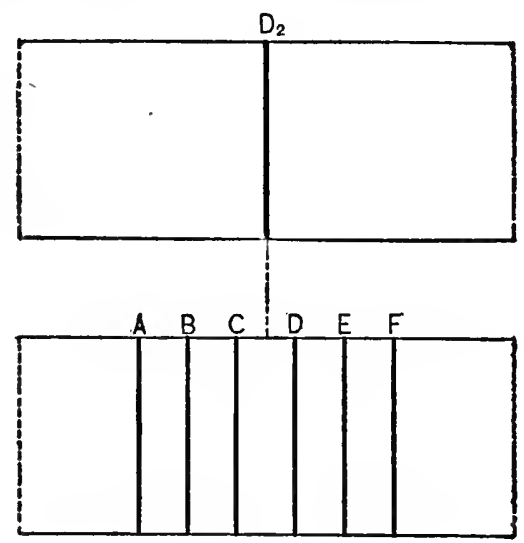

FIG. 4.

in this direction, there exists at every instant an electromagnetic force analogous to that which would act on a short portion of an electric current coinciding in direction with the velocity of the particle. This force, therefore, will be directed along the radius $O A$, passing through the moving particle,

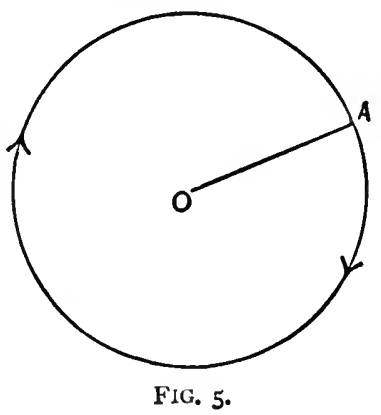


and will act, as the case may be, either from $A$ toward $O$, or in the opposite direction. The effect of this new force, which augments or diminishes the force which maintains the particle in its orbit, is to vary its vibratory period, - that is, the time required for the particle to describe the circumference, - just as a change in the intensity of the force of gravity has the effect of varying the period of oscillation of a pendulum.

From the effect produced by a magnetic field on a circular vibration we may easily pass to that produced on any vibration whatsoever by a consideration like the following :-

Light vibrations are perfectly well understood. They follow the same laws as do the small oscillations of a pendulum, and are, in general, elliptical; in special cases they may be rectilinear or circular; they are always transverse, that is, they lie in the plane perpendicular to the light ray. Now it may be shown that every elliptical vibration is kinematically equivalent to the resultant of 
two circular vibrations of opposite directions of rotation, the one right-handed (motion in the same sense as that of the hands of a watch), the other lefthanded; and in addition the circular vibration which has the same direction of rotation as the ellipse $E F$ (Fig. 6), namely $A B$, has a diameter equal

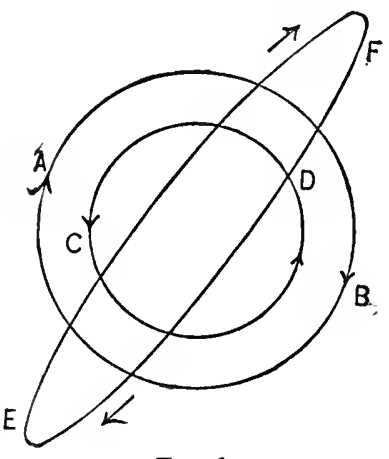

FIG. 6. to half the sum of the axes of the ellipse, while the other circular vibration, $C D$, has a diameter equal to half the difference of the same axes. ${ }^{1}$. If we do not wish to have

1 Employing the usual symbols, the elliptical vibration, referred to its axes, may be represented by means of its rectangular components

$$
x=a \sin \theta, \quad y=b \cos \theta .
$$

This is evidently equivalent to the resultant of two circular vibrations, one of which is right-handed like the given ellipse, and has the components

$$
x=\frac{a+b}{2} \sin \theta, \quad y=\frac{a+b}{2} \cos \theta,
$$

while the other is left-handed and has the components

$$
x=\frac{a-b}{2} \sin \theta, \quad y=-\frac{a-b}{2} \cos \theta .
$$


recourse to a mathematical demonstration, we may convince ourselves of the proof of this statement by employing a special piece of apparatus which serves, among other pur-

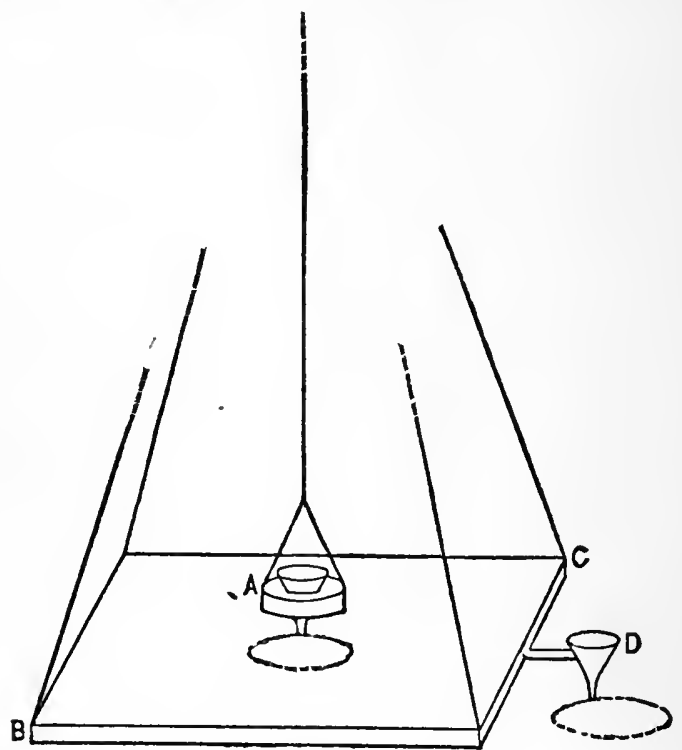

FIG. 7.

poses, that of effecting the composition of two pendular vibrations of circular character (3).

Two pendulums (Fig. 7) are suspended from two fixed points, which for the sake of simplicity are not represented in the figure, 
placed one above the other on the same vertical line. One of the pendulums consists simply of a wire carrying at its lower end a heavy ring and a funnel, $A$, filled with sand; the lower part of the other consists of a platform $B C$, situated below $A$, which carries on one of its sides a second funnel, $D$, also filled with sand. The length of the first pendulum may be varied at will, but for the experiment with which we have to deal this length should be such that the two pendulums have the same period of vibration. A simple electric device controls the openings of the funnels and thus prevents or allows the flow of sand.

At first let us suppose the pendulum $B C$ to be fixed while we impart a circular motion to $A$; it is easy to ascertain whether or not this motion be circular from the trace left by the sand on the platform $B C$. Let us then impart a circular motion to the pendulum $B C$ as well, but in the direction opposite to that of the first pendulum; we may easily ascertain if we succeed in doing this by observing the trace left by the sand from the 
funnel $D$ on the plane of support. If, now that the two pendulums are vibrating, we allow the sand to run out of the funnel $A$, it will form an elliptical trace on the platform $B C$. This ellipse becomes a straight line if the two component circular vibrations have equal diameters. We can thus convince ourselves of the truth of the statement made, and in addition we learn that, when the two component circular vibrations of opposite rotation have equal amplitudes, the resulting vibration is rectilinear.

Returning now to the case of the particle vibrating in a magnetic field, it in general executes an elliptical vibration, for which we may conceive the two equivalent circular vibrations to be substituted. But these last are of opposite sign of rotation; if one of them is accelerated by the magnetic field, the other must be retarded. As soon as their periods cease to be equal, they can no longer cause a single spectrum line, but cause instead two new lines situated on either side of the single primitive line. This explanation, furnished by Lorentz's theory 
to explain the experiment of Zeeman, was proved correct by this able experimenter in a series of new experiments, which showed that the two new lines were in fact due to circular vibrations, one right-handed and the other left-handed.

Suitable qualitative and quantitative experiments made it possible to deduce two very interesting results from the Zeeman phenomenon. On investigating which of the two new lines was due, for a given direction of the magnetic field, to the righthanded vibrations and which to the lefthanded, the sign of the charge of the vibrating particles could be determined, and it was recognized that, in order to make the observed facts accord with their explanation, it was necessary to admit that these particles possessed a negative rather than a positive charge. In the second place, it was possible to obtain an approximate evaluation of the ratio of the electric charge of the vibrating particle to its mass. The result to which this led was, that this ratio is more than a thousand times greater than that which re- 
lates to the atom of hydrogen in electrolysis, and hence still greater than that pertaining to the atoms of other substances.

This result may be interpreted in several ways, the more important of which are the following: either the vibrating particles are ions, and the charge of each is more than one thousand times as great as that which pertains to each valency in electrolysis; or the vibrating particles have a charge equal to that of the electrolytic ions, and their mass is less than one-thousandth as great as that of an ion of hydrogen. The second interpretation is naturally the one accepted, and the vibrating particles are considered to be free electrons. These therefore possess, or at least there is united with them, a small material mass; but we shall see that this same mass probably has an electromagnetic cause. At any rate, this result is corroborated by those which are reached in other ways, as will be shown later.

Lorentz's theory receives, therefore, a splen-. did confirmation through the experiments of Zeeman; and hence it may be retained, that 
the structure of the material atoms is such as to permit the negative electrons, which form a part of them, to vibrate freely, while the positive part remains relatively fixed. Thus we conceive a neutral atom to consist of one portion which in the aggregate has a positive charge, and of one or more negative electrons, which move about it like the satellites about a planet, held in their orbits by the electric force.

The so-called oscillators, or the apparatus used to generate electromagnetic waves, have recently come into considerable prominence. One of the possible but impractical forms consists of an electrified body executing vibratory motion; for example, one driven by a sonorous body in vibration. Now if one imagines the electrified particle to be replaced by a simple electron, and if one supposes the period of vibration to be so small that it would be expressed by a fraction whose numerator is unity and whose denominator is a number of fifteen places, then this electron would generate ordinary light waves instead of Hertzian electromagnetic waves. 


\section{CHAPTER III}

NATURE OF THE CATHODE RAYS (4)

THE phenomena on which we shall now touch show the negative electrons in the act of undergoing very rapid motions of translation instead of the vibratory motions which we have considered in the preceding chapter. Hence they present themselves under conditions favourable for a closer study, and thus, by modifying their motion in various ways, new and interesting effects may be produced. But for the sake of clearness it will be well first to state the principal characteristics of electric discharges in rarefied gases.

Let us consider a glass tube, $A C$ (Fig. 8), through the end walls of which are fused two platinum wires terminating in aluminium electrodes, $A, C$. If the air pressure in the tube is somewhat less than that of the atmosphere, - for example, eight or ten millimeters of mercury, - and if an electric discharge is 

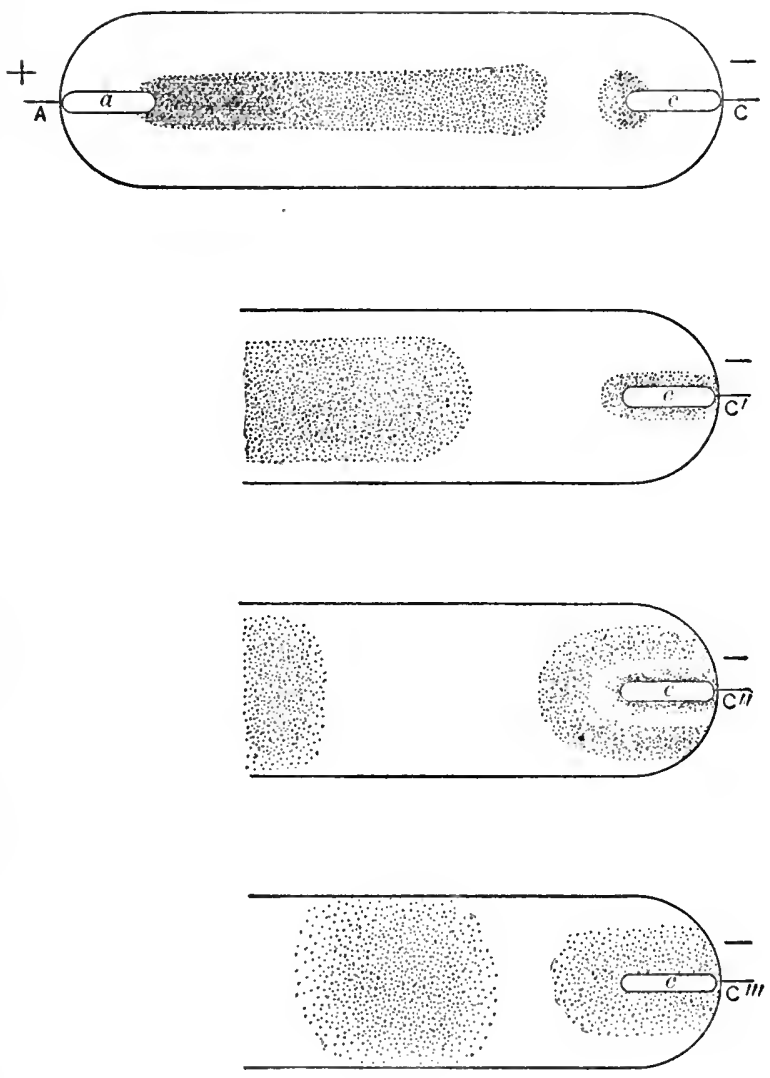

FIG. 8.

caused to pass from one electrode to the other, instead of the well-known loud and brilliant spark which is formed in the open air, a char- 
acteristic luminous phenomenon is obtained, in which two regions are distinguishable: the positive luminous column, a sort of ill-defined, rose-coloured spark having a blurred contour which reaches from the anode up to within a short distance of the cathode; and the negative column, or negative glow, violet in colour and contiguous with the cathode. Between these two luminous regions there is an interval called the Faraday dark space.

If now the pressure of the air is diminished, the luminous character changes. We will concern ourselves with the negative light without considering further the positive column which, with increasing rarefaction, gradually diminishes both in size and in luminous intensity, often subdividing into distinct regions separated by relatively dark intervals (striated discharge). At first the negative light extends over the entire cathode, as at $C^{\prime}$, in case initially it only covered the extremity; but later, with still further rarefaction, it extends all around to greater and greater distances, detaching itself at the same time from the electrode, as at $C^{\prime \prime}$. In the meantime a 
'new luminous stratum forms in contact with the electrode, and thus the negative light is divided into two parts; namely, the first negative column, adhering to the cathode, and the second negative column, or negative glow, separated from each other by a relatively dark region which, to distinguish it from the Faraday dark space, is called the cathode dark space. Continuing the rarefaction still further, the two luminous negative columns extend farther and farther out, becoming continually less bright and sharp in outline ( $C^{\prime \prime \prime}$, Fig. 8). The interval which separates them also becomes greater; and, when the highest rarefaction is obtained, that is to say when the air pressure is reduced to less than one-thousandth of a millimeter of mercury, almost every trace of luminosity in the gas disappears.

But before this stage is reached a new phenomenon appears. At first the portion of the walls of the tube about the cathode, and later that in front of it, becomes luminous, diffusing a brilliant light, usually green, due to a species of phosphorescence, 
or perhaps better of furorescence, since this is the name given to the emission of light by fluorspar and certain other substances, which does not last appreciably after the cause which produced it has ceased. The cause of this phenomenon is to be sought in the cathode, because, if an obstacle is placed between it and the wall, a very sharp shadow is thrown, as if the fluorescence were excited by invisible radiations sent out from the cathode. We will now turn our attention to these radiations, which are called Cathode Rays.

They are propagated in straight lines and leave the cathode in a direction at right angles to its surface; hence if this has the form of a concave mirror, the cathode rays converge practically to the centre of curvature. When concentrated in this manner their singular properties become more evident, as Sir William Crookes has shown in a very brilliant and suggestive manner with the aid of cleverly devised apparatus.

The principal properties of the cathode rays are the following: they excite phospho- 
rescence not only in glass, as we have seen, but in a large number of other bodies, including those which phosphoresce under the action of light. The cathode rays heat bodies which they strike and tend to move them as if the impact were mechanical. It is possible, however, that this mechanical action may be, at least in a large part, a simple consequence of the preceding effect. Finally, bodies struck by cathode rays become sources of new radiations; namely, the famous X-rays discovered by Professor Röntgen. In order to explain all these phenomena, Crookes brought forward his hypothesis of radiant matter.

As early as I 8 I 6 the celebrated Faraday (5) pointed out the possibility of a fourth state of matter, as a consequence of a hypothetical transformation which transcends evaporation by as much as evaporation transcends the fluid state; or, he expressed his thought still better by saying that he looked forward with the greatest impatience to the discovery of a new state of the chemical elements. He suggested further, and this has an especial 
interest with reference to the theory with which we are now occupied, that the decomposition of the metals, their recomposition and the realization of the formerly absurd idea of transmutation, were problems which chemistry one day must solve.

According to Crookes, when the electric discharge takes place in a highly rarefied gas, very minute negatively electrified material particles are projected from the cathode and, forming a fourth state of matter transcending the gaseous state, produce the observed effects as a result of their collisions; moreover, the trajectories of these particles constitute the cathode rays. It was later thought that the particles were the actual atoms of the gas residue which on account of its extreme rarefaction presented such new properties as were brought to light by the rotation of the vanes of Crookes' famous radiometer.

But some people, among whom was the illustrious Hertz, preferred to consider the cathode rays as an undulatory phenomenon similar to light, having its origin at the sur- 
face of the cathode and its seat in the ether. However, this opinion had very soon to be given up on account of subsequent experiments. Thus, while many physicists, notably J. J. Thomson (6) in England, to whom we owe so much of the present electron theory, and $Q$. Maiorana (7) in Italy, were finding out that the velocity of the cathode rays is noticeably less than that of light, J. Perrin (8) was making it evident that the cathode rays produce a transport of negative electricity. This last effect may be obtained even when the rays have passed through a thin metallic plate, as was later shown by Lenard (9).

Perrin's experiment may be made with a discharge tube similar to that shown in Figure 9. The cathode consists of an aluminium disk, and the anode $A B D E$ is a cylindrical box with circular openings at the centre of the bases. This box is in connection with the earth, and contains the conductor $F$, which is connected to an electroscope. The conductor $F$ usually has the form of a hollow cylinder with an opening turned toward 
36 NATURE OF THE CATHODE RAYS

that in the base, $D E$, of the anode. A negative charge collects on the conductor $F$ when the discharge enters the tube. Evidently this can only be due to a transport of charge effected by the cathode rays. Moreover, at the approach of a magnet, the action of which, as we shall see, is to make the cathode rays assume a curved path, the rays cease to

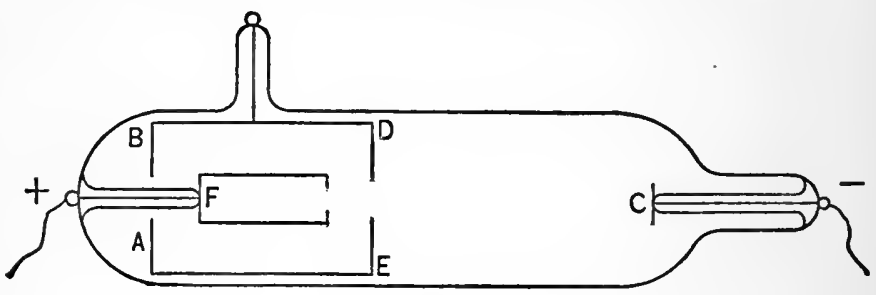

FIG. 9.

enter the cylinder $A D$ and cause a luminous spot to appear on the base $D E$, which for this purpose is usually coated with a phosphorescent substance. Now just as soon as the conductor $F$ ceases to receive the rays, it, in turn, ceases to receive electricity.

Naturally the discovery of these facts furnished the strongest support for Crookes' theory. But countless recent experiments due to many physicists have led to a slight 
modification, and a better statement of the original hypothesis and to the admission that the particles, which in their rapid motion constitute the cathode rays, can be nothing but the negative electrons themselves. This opinion, at present held by all, rests principally on the following facts which have been accurately verified, and with which we shall concern ourselves in detail a little later. In the first place, the cathode rays always have identical properties whatever may be the rarefied gas in which they are formed, and whatever may be the nature of the cathode; in the second place, the moving negative particles all possess that same small mass less than one-thousandth that of a hydrogen atom which is encountered, as we have seen, in the study of the Zeeman effect, and which is deduced from the results of various other phenomena as well.

Cathode rays may also be produced without having recourse to the electric discharge. Thus, for example, a body exposed to the action of light, or better of ultraviolet rays, emits electrons. Unless the surrounding 
gas is extremely rarefied they unite with neutral atoms and form negative ions; but if the gas is almost entirely removed, the electrons remain free, and on leaving the body form true cathode rays (ro), which in general possess a velocity less than that which they have in discharge tubes. This velocity decreases as the negative potential of the illuminated body is diminished.

Also in the case of the cathode rays the mass of the electron was not separately determined, but rather the ratio between the electric charge of any electron and its mass. Such a determination as this is based on the effects produced on the cathode rays by electric or magnetic forces, and these effects are in good accord with the accepted hypothesis. In fact, it is clear that when an electric force acts on negative particles in motion, they should deviate from their ordinary rectilinear path; and since an electrified particle in motion should behave in a manner analogous to a current, or more accurately to an element of current, it follows that the particle itself should deviate from its ordinary 
path when it is exposed to the action of a magnetic field. But we shall consider such phenomena and the measurements relating to them a little farther on. 


\section{CHAPTER IV}

THE IONS IN GASES AND IN SOLIDS

In electrolytes the electrons are joined to the neutral atoms to form free ions, and the motion of these ions is what constitutes the electric current. At present the opinion is held that the same thing happens in gases; namely, that when a gas possesses electric conductivity, it owes it to the presence of ions, and to their motion under the action of electric forces. The hypothesis of the ionization of gases, which, for a long time, was held by very few, is now generally admitted in consequence of the numerous experiments made in recent years.

We are, then, of the opinion that a gas contains free ions. These are ordinarily present in such a small number that the resulting conductivity is very small. But there are circumstances in which, by the 
action of appropriate external energy, the gas is ionized; that is to say, many of its atoms are broken up into positive ions and negative electrons. If the gas is not sufficiently rarefied, the electrons unite with the neutral atoms and form negative ions. Moreover, certain facts seem to indicate that atoms or neutral molecules can unite with ions to form groups which, while having the usual charge of the ions, possess masses much greater than those which can pertain to a simple ion.

The most natural explanation of the known facts, and in particular of those about to be mentioned, is that the electrical conductivity of gases is due to the presence of electrified particles which are free to move between its molecules.

An ionized gas loses its conductivity when passed through minute interstices, say through a mass of glass wool, or through long and fine metallic tubes, or is made to bubble through a conducting liquid (I I), which, however, should not contain any radio-active substance. The same result is 
obtained if the gas is made to pass between two oppositely electrified conductors in such a way that it may serve as a conductor for the current. In the first case, the phenomenon is explained by the attraction exerted on the ions by the bodies near which they pass; in the second case, the two conductors attract and hold the ions which carry a charge opposite in sign to their own and so remove them from the gas.

The manner in which an ionized gas behaves when it is carrying an electric current is also in perfect accord with the accepted hypothesis. Let us suppose that we have, for example, two parallel metallic disks, one of which communicates with the insulated pole of a battery, and the other with an electrometer. If we ionize the air between the disks by passing Röntgen rays through it, and if we vary the value of the potential furnished by the battery, we find that the gas fails to follow the well-known law of Ohm, which holds for constant electric currents, and according to which the intensity of the current in a conductor in- 
creases in proportion to the difference of potential between its ends. In fact, the intensity of the current measured by the charge, which is acquired in a given time by the disk in communication with the electrometer, increases considerably less rapidly than the potential. The intensity even finally assumes a limiting value which does not increase as the potential of the battery is raised. When the current has attained this value, called the saturation value, all the ions generated in a given time by the Röntgen rays (or in general produced by whatever source of ionization is employed) are utilized in transmitting the current in this same time. An increase in potential is of no effect, as there are not a greater number of ions to be disposed of.

Moreover, a curious phenomenon met with by the writer (12), and which was confirmed and rightly interpreted by J. J. Thomson and E. Rutherford, is obviously explained by the accepted theory. The phenomenon is the following: if the distance between the two metallic disks considered 


\section{THE IONS IN GASES AND IN SOLIDS}

above is varied, the intensity of the current which traverses the ionized air between them varies as well, but in a manner contrary to that which one would suppose. In fact, the intensity of the current increases, within certain limits, with an increase of the distance. This is easily explained when we reflect that with an increase in the distance between the plates there is an increase in the amount of air in which the phenomenon takes place, and in consequence also in the number of ions, which, by their motion, constitute the saturation current.

The ions in gases move around between the molecules, frequently colliding with them. New ions may form by the breaking up of neutral molecules, and ions of opposite sign may recombine into molecules. This last action, namely, the disappearance of ions, is continually taking place, and it is because of this that the number of ions does not increase beyond a certain limit under the action of an ionizing cause.

If the ions are generated in a single region of the gas, they diffuse into the remaining 
portion. In gases under ordinary pressure the velocity of diffusion is usually extremely small because of frequent collisions; but if an electric field acts, the velocity of diffusion becomes large; the first time that a measurement of this kind was made ( 13 ) the velocity was found to be several decimeters per second:

Ultraviolet rays, cathode rays, Röntgen rays, rays emitted by radio-active substances, heating to a relatively high temperature, are all causes of ionization. This is greater or less according to circumstances, and is limited, as has already been mentioned, by a continual recomposition of atoms and neutral molecules.

But there exists still another cause of ionization, to which in reality some of the above causes reduce; this is the collision of the ions (and, in fact, of the electrons as well, since probably some of these exist, at least momentarily, in the free state in gas under ordinary pressure) with the atoms and molecules. When an ion possesses a sufficiently high velocity, it can furnish the energy neces- 
46 THE IONS IN GASES AND IN SOLIDS

sary to transform an atom into a positive ion and a negative electron, and hence also to transform a molecule into two ions of opposite sign. Let us briefly take up these various means of ionizing a gas.

Light radiations, and especially the ultraviolet, may ionize a gas in two different ways. If they strike a solid or liquid body, they produce an emission of negative electrons which results in the rapid discharge of the body, if it was negatively electrified, and even the formation on it of a positive charge, as has been demonstrated by the writer (14). Ordinarily the experiment is performed with metals, because the effect is rather weak with liquids, and solid insulators are not so well adapted to quantitative determinations. As a source of active radiations, the invisible ultraviolet rays emitted by an arc light or by an electric spark are employed, although certain bodies, such as the alkaline metals and amalgamated zinc, give a marked effect even with visible radiations. Now if the electric field determined by the negative charge of the body is sufficiently intense, 
the negative electrons which are emitted may acquire a velocity great enough to ionize the neutral atoms by impact.

But even directly the more refrangible ultraviolet radiations given off by the electric spark cause ionization of the gas through which they pass, as was demonstrated by Lenard (I 5), who allowed the radiations from a spark formed between aluminium wires to fall on electrified bodies. These became discharged with about the same rapidity whether they were charged positively or negatively and whatever was the nature and condition of their surfaces. All this could not be attributed to a surface action, but rather to an action on the mass of the air traversed by the radiations; that is, to the ionization which they produced. An experiment which may be repeated also with other sources of ionization confirmed this explanation. It consists in blowing the air from the place where it is ionized to another place, where, as a result of the conductivity which it retains for a certain time, it brings about the discharge of electrified bodies. In order 
to cause the effect to cease it is sufficient to intercept the radiations.

It appears that only the more rapid ultraviolet vibrations produce direct ionization of gases in any marked degree. Indeed, the experiments described above do not succeed except when the path traversed in the air by the radiations is reduced to but a few centimeters, and it is a well-known fact that the most refrangible ultraviolet radiations are very rapidly absorbed by air at ordinary pressure.

The cathode rays which are, as we have seen, nothing but negative electrons in motion, ionize a gas, as will be explained before long in some detail.

With regard to the Röntgen rays, which apparently are the manifestation of ether waves generated by the sudden variations in velocity of the electrons, the ionization of gases produced by them seems to be due to a sudden electric impulse produced in the electrons of the gaseous atoms.

Finally, a rise in temperature, which is equivalent to an increase in atomic velocity 
and apparently to an increase in the velocity of the negative electrons as well, naturally tends to free the latter from their bond with the positive part of the atom. A red-hot metallic wire ionizes the gas in contact with it, and gases in flames always appear to be strongly ionized.

In order that the molecules of the gas may become ionized by the impact of those ions which already exist in it, it is generally necessary to expose the gas to the action of sufficiently intense electrical forces. In too weak a field, the ions, while they follow the electric force, do not acquire a sufficient velocity between one collision and the next, and the effect of the collisions is to keep this velocity constantly at a low value, since naturally a part of the energy of motion of the ions is given up to the molecules which have been struck. Under such circumstances the paths described by the ions cannot differ much from the lines of electric force, or, in other words, the ions must continually move very nearly in the direction of the force which urges them. The so-called phenomena of 
the electric shadow and similar ones of which the reader will find a partial treatment in another place (I6), are the immediate consequence of this.

But if a sufficiently strong electric field acts on the gas, ionization by impact takes place, and it is on this phenomenon that a satisfactory explanation of the complex and varied phenomena of the electric discharge is at present founded. In order to take this matter up, we would be obliged to go beyond the limits assigned to this chapter; but in view of that which follows, it will be useful to call attention by way of an example, to the explanation which is given of the formation of the two columns of negative light and the dark space included between them in the case of the electric discharge in highly rarefied gases.

The phenomenon is started by the few electrons existing in the gas, or perhaps also by the negative electrons expelled from the cathode. These electrons move with accelerated motion and rapidly acquire a velocity sufficient to make them capable of ionizing 
by impact the gas molecules at some distance from the cathode. This gives rise to the second negative column, or negative glow, which is thus a region of the gas where ionization takes place. The electric force drives the positive ions created in this manner toward the cathode, close to which they possess the velocity required to ionize the gas molecules. This causes the formation of the first column of negative light.

The electrons produced in this region move away from the cathode, and in this way the two regions of ionization furnish each other the necessary ions or electrons. The cathode dark space is thus simply the region traversed by the electrons constituting the cathode rays, and especially by the positive ions which move toward the cathode, before they have acquired the velocity necessary to produce ionization.

We will not concern ourselves further with the negative electrons after they have arrived at the second negative column; however, it is of interest to us to know what becomes of the positive ions after they arrive 
52 THE IONS IN GASES AND IN SOLIDS

at the cathode. Some of them naturally become neutralized by the negative electrons; but others, on account of their velocity, which varies in direction as a result of collisions, may bend around the cathode, or even pass through it, if it is provided with openings or canals, or if it is made of wire gauze. Beyond the cathode the positive ions then constitute the positive or anode rays, analogous to the cathode rays, and which are often called canal rays, the name given them by Goldstein.

An electric or a magnetic field causes a deviation of the positive rays, but in the direction opposite to that which would be observed with cathode rays; it is precisely on account of this that the conclusion is reached, that these rays consist of positively electrified particles in motion. However, the deviation is noticeably smaller for the positive rays than for the cathode rays under similar conditions. From measurements of the deviation it may be shown that the particles in motion do not possess a minute mass as in the case of the cathode rays, but a mass 
comparable with that of atoms or of electrolytic ions. In this case, therefore, we have to deal not with positive electrons, but with ions and probably with groups of greater mass as well.

If we admit electric conductivity to be a phenomenon of convection in gases as well as in liquids, the analogous hypothesis for solid conductors, already stated in Chapter I, becomes all the more natural. And, inasmuch as it appears that only the negative electrons, and not the positive, can exist isolated, it is held that the electric current in a conductor consists, at least principally, in the motion of negative electrons. The experimental evidence that metals do not offer an insurmountable obstacle to the motion of the electrons is furnished by their perviousness to the cathode rays. Without entering into details we may add that this mode of considering the current permits us to explain fairly well various observed facts, as, for example, the proportionality between the thermal and electric conductivity of various bodies, and to explain such phenomena as those relative to 
54 THE IONS IN GASES AND IN SOLIDS

the optical properties of metals. Thus the electron theory not only finds no contradiction in phenomena of this sort, but also shows itself able to furnish a simple explanation of them. 


\section{CHAPTER V \\ RADIO-ACTIVITY}

THE discovery of the so-called X-rays by Professor Röntgen early in 1896 gave rise to numerous experiments relating to the possible existence of other radiations capable of affecting photographic films and of passing through opaque bodies. Effects of this sort were described by Le Bon; but we will not consider these, as it was recognized that the effects attributed by this experimenter to a new radiation which he called "black light" proceeded, at least in nearly every case, from causes which have no intimate relation with the subject which we are now about to treat.

Certain experiments which have, on the other hand, a relationship with radio-activity were made by Henry (I 7) with phosphorescent sulphide of zinc; by Niewenglowski (I8) with calcium sulphide; and by Becquerel (19) 
with double sulphate of uranium and potassium. The result of these experiments is that these substances emit rays capable of passing through opaque bodies and acting on a photographic film, when phosphorescence is excited in them by exposure to light or to X-rays.

When Becquerel began his experimentation, he had a special object in view. It was already known that the X-rays had their origin at that part of the wall which is rendered luminescent where the cathode rays strike; it was consequently natural to suppose that phosphorescence and the emission of X-rays were related phenomena, though later it was recognized that such is not the case. H. Becquerel therefore wished to determine whether bodies rendered phosphorescent by the action, not of cathode rays, but of light, would emit X-rays. And since these rays can affect a photographic film even when it is surrounded by opaque objects, the French physicist placed various bodies above a film, protected in this manner from the light, and exposed the whole 
to sunlight. After several unsuccessful attempts, he obtained a marked effect with crystalline laminæ of the double sulphate of uranium and potassium, since on developing the film he saw an image of the laminæ and the shadow of a silver coin which had been placed below one of them. It seemed, therefore, that the phenomenon he had predicted had really taken place. But Becquerel happened also to obtain the same effect with poor illumination on a cloudy day, from which he suspected that the effect itself did not depend on the action of the light. And, in fact, he very soon proved (20) that the uranium salt continually and spontaneously emitted rays able to go through opaque bodies and to act on photographic films without the necessity of its being exposed to the light.

Further research showed (2I) that the rays from the uranium salt share with the X-rays, not only the property of traversing opaque bodies, of acting on photographic films, of rendering phosphorescent bodies luminous, and, as was later shown, the negative prop- 
erty of not being susceptible of reflection and refraction, and hence of polarization, but also another property which was recognized shortly after the X-rays were discovered (22); namely, that of ionizing gases through which they pass. A method of studying the Becquerel rays, which is more rapid than the photographic method, is based on this property; it consists in the measurement of the velocity with which an electrified body becomes discharged when the surrounding gas is exposed to the action of the rays.

For this purpose we may make use of any electrometer connected to a metallic disk placed at a short distance from a second disk; and we may experiment in two ways. Either the second disk is put in connection with the earth and then one notes the rate of diminution of an electric charge communicated to the electrometer when the air between the two disks is ionized by the radio-active body; or the second disk is charged and the rapidity with which the electrometer deviates is observed. With very active bodies a galvanom- 
eter may be employed, but if bodies of weak radio-activity are to be investigated, it is better to use a gold-leaf electrometer, or better still, an electroscope with but a single leaf consisting simply of a vertical metallic rod, $A B$ (Fig. Io), at the upper end of which a very light gold or aluminium leaf, $C D$, is fastened. In order to secure good insulation the rod is supported by a small piece of sulphur, $S$. The electrical capacity of the conducting system $A B C D$ is extremely small, and hence it results that the diminution in the divergence of the leaf $C D$ is not too slow. The electroscope becomes an electrometer,

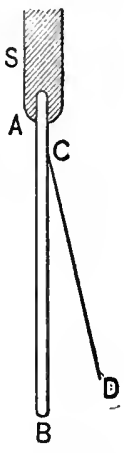

FIG. IO. if the position of $C D$ is observed by means of a microscope and ocular scale. The potential corresponding to each division of the scale may be determined in advance by the use of a battery of small accumulators.

The writer prefers a form of electrometer slightly different from that which has become classic for the study of feebly radio-active bodies. The sulphur or fused quartz insu- 
lator $S$ (Fig. I I) is quite slender and held with mastic or gutta-percha to the bottom of a small bell-shaped piece of metal connected with the $\operatorname{rod} A B$. This form of support obviates or reduces the creeping of the charge from the rod to the surface of the s. insulator. Moreover, the writer in again adopting an arrangement which he devised many years ago, uses an ordinary millimeter scale placed at several meters' distance from the electrometer, instead of one annexed to the ocular. A converging acromatic lens forms a real FIG. rr. image of the scale in the plane in which the metallic leaf moves, and thus both the leaf and the scale are seen simultaneously in the field of the microscope.

In one of the electrometers lately constructed by the writer the rod and the gold leaf have scarcely one-fourth the dimensions shown in Figure I I. It is particularly well adapted to the demonstration of radio-activity, since it is so sensitive that, when one of the uranium salts is approached in such a way 
that the rays emitted by it pass through the thin aluminium walls of the electrometer case, the gold leaf, when previously electrified, may even with the unaided eye be seen to droop.

As more and more experiments were made, it was established that all the compounds of uranium are radio-active, that is, they emit Becquerel rays, and their activity is in proportion to the quantity of uranium which they contain; this shows that radioactivity is a property of the uranium atom and one which remains unaltered when the atom itself enters into combination with the atoms of other chemical elements.

Thorium, as well as uranium, is radioactive, to a slightly different degree, as was independently established by Schmidt (23) and by Mme. Curie (24).

If it had not been for the discovery of certain bodies whose radio-activity is hundreds and even thousands of times greater than that of uranium, it would have been perhaps very difficult to have studied radio-activity in a thorough manner and to have determined 
the peculiarities and the probable original cause of this interesting phenomenon, or, at least, all this would have required much time and most accurate research.

M. and Mme. Curie chanced to find certain specimens of chalcolite and pitchblende (in particular that which is mined at Joachimsthal), which were somewhat more active than pure uranium. Recalling that radio-activity is an atomic property, the phenomenon could not be attributed to uranium contained in these minerals, and it was necessary to suppose that an unknown substance, more active than uranium itself, was present. Having recourse to physical and chemical methods of separation, certain compounds of bismuth were extracted from these minerals having a radio-activity as much as four hundred times that of uranium (25). The name polonium was given to the unknown substance contained in these compounds, the radio-activity of which diminishes slowly as time goes on. Later the Curies and M. Bémont (26) extracted from pitchblende a small quantity of a very active body, chemically analogous to barium 
and associated with it in almost every reaction, to which they gave the name radium. Another radio-active body associated with thorium and possessing similar chemical properties was discovered by M. Debierne (27), who called it actinium.

Various other investigators have succeeded in deriving still other noticeably radio-active substances from many different minerals, but especially from pitchblende; the nature of these substances is not yet well understood. In particular Elster and Geitel (28) obtained a rather radio-active sulphate of lead which seems to contain simply radiferous barium. Nor is the radio-active lead obtained by Giesel (29) any better characterized, while that found by Hofmann and Strauss (30) would, in some respects, seem to resemble polonium. The active lead, or radio-lead of these authors, exhibited one property worthy of mention. Under certain conditions the active sulphate of lead loses a large part of its radio-activity, which it later slowly recovers; but if it is exposed to the bombardment of the cathode rays, its radio- 
active properties are fully restored in a few minutes.

Recently Hofmann and Wölfl (3I) have partially separated the active substance from the inactive lead, and have determined that it differs from the polonium of Mme. Curie in the constancy of its radio-activity.

The substance called radio-tellurium by Marckwald (32) also resembles polonium in its properties, the only difference being that its radio-activity does not seem to diminish with time. It would seem that radio-tellurium is the body to which the activity of the bismuth extracted from the Joachimsthal pitchblende is due, and it is considered by this author to be a new body belonging to the sulphur and tellurium series, because it is deposited on sticks of bismuth or antimony introduced into an acid solution of active chloride of bismuth. Marckwald obtained in this manner about six decigrams of quite active material from 850 grams of the salt. An active bismuth quite similar to that of the other investigators was discovered by Giesel (33), who later separated from it a prod- 
uct identical with that obtained by Marckwald. Finally a supposed new radio-active element was separated from thorium by Baskerville, who called it carolinium (34).

As we see, a great uncertainty still exists regarding the nature and even the separate existence of the greater part of the radioactive substances; so much so, in fact, that there are some who consider the effects attributed to Mme. Curie's polonium as due to an induced radio-activity, that is, to a transitory phenomenon, of which we shall speak later; there are others who believe radioactive thorium and actinium to be identical. The chemistry of the radio-active bodies is only in its initial stages, and at present radium, whose characteristic spectrum is now known, is the only substance the existence of which, as an element distinct from the others, seems to be sure.

Radium has never been prepared in the free state, but, nevertheless, several of its salts are known. For example, the small quantity of radio-active substance, which the Curies were first able patiently to obtain from 
several tons of pitchblende residues, remaining after the uranium had been extracted, was the chloride of radium. These residues contained compounds of almost all the metals, among which were barium, bismuth, and the metals of the rare earths. Chemical processes, which it would take too long to describe here, permit the extraction of the barium with the radium, the bismuth with the polonium, and the rare earths with the actinium; after this there remains the separation of each of the radio-active bodies from the body which accompanied it, in spite of previous chemical reactions. This separation, to which Mme. Curie has devoted many years of labour, has not yet been completely successful, except in the case of radium, where the following process was employed.

The radiferous chloride of barium, of which about eight kilograms is extracted from a ton of pitchblende residues, is dissolved in hot water, so as to form a saturated solution. On cooling, crystals, which we will call $A$, are thrown down, while on evaporating the remaining solution, another 
chloride, which we will call $B$, is obtained. Since the chloride of radium is slightly less soluble than the barium chloride, it results that the chloride $A$ is richer, and the chloride $B$ is poorer, in radium than the chloride used in forming the solution. This may be ascertained by determining the relative radio-activity. A second operation exactly similar to the above is carried out with each of the products $A$ and $B$; now since the least active part obtained from $A$ and the most active part obtained from $B$ are about equally radio-active, they are reunited, and thus the four portions which result from the second operation reduce to three of different richness in radium. The process is continued in the same manner; however, to prevent an inordinate increase in the number of portions, no use is made of those whose radioactivity is very small, and the operation is discontinued on those portions whose radioactivity is sufficiently great. It is also found advantageous to make use of the mother liquor obtained from one operation to dissolve the crystals obtained in the succeeding 
operation. When the greater part of the inactive substances is eliminated by this process, it is carried on still further, but now the least active parts are more freely discarded. In this way a small amount of radium is lost, but the purification proceeds more rapidly, to accomplish which it is advisable to acidulate the solvent more and more with pure hydrochloric acid. Finally, practically pure chloride of radium is separated to the amount of two or three decigrams per ton of the residues employed. At present bromide of radium is also prepared in the pure state.

Besides the measurement of the degree of increasing radio-activity a spectroscopic examination of the product may be employed to estimate its purity, since radium possesses a spectrum which perfectly characterizes it and which was studied by Demarçay. If an impure product is examined, the spectrum of barium appears with the spectrum of radium; but as the process of purification goes on, the barium lines become weaker and finally almost completely disappear.

After the principal effects due to radio- 
active bodies were discovered, the attention of physicists was turned to the direct study of the rays emitted.

All known radiations are, or at least we conceive that they are, of two sorts; those due to waves propagated in the ether, and those due to the motion of electrified material particles. Not only the luminous rays properly so called, and the invisible heat and ultraviolet rays, but also, as is thought, the Röntgen rays belong to the first sort. To the second sort belong the cathode rays, which are, in fact, considered to be due to the motion of negative electrons.

It is not difficult to decide on the nature of new radiations which belong to one or the other of these two categories; in fact, while an. electric or magnetic field cannot in the least degree modify the form of luminous or of Röntgen rays, etc., they should cause a marked curvature of the path described by electrified particles, unless the velocity is exceedingly great. However, there exists, as it appears, certain new radiations to which their discoverer, Blondlot, has given the name 
of n-rays (35), which seem to possess some of the properties of heat rays, but which appear to possess other very strange properties. We shall not attempt to describe them here, since their nature is still enigmatic.

In order to acquire a clear conception of the nature of the rays emitted by radio-active bodies, it was necessary to subject them to magnetic or electric forces, and therefore to place the radio-active bodies either between the poles of a powerful magnet or between two metal plates oppositely electrified. Moreover, in order that the possible deformation of the rays might be recognized, they had to be passed through a small opening or diaphragm, on the far side of which either a phosphorescent body or a photographic plate protected from the light by a black paper covering was placed. With the phosphorescent body the displacement of the luminous spot is at once seen when an electric or a magnetic field deforms the rays, and the same result is detected when the plate is developed. It is generally preferable to use the photographic plate, because the length 
of exposure, which may be as great as is desired, eventually compensates for the low intensity of the radiations which are studied.

Applying these methods, it was recognized from the very first that in general a radioactive body emits rays which are deviated by the magnetic and the electric field, and at the same time other rays which are not deviated. It was further determined that the former behave in all respects like cathode rays of high velocity, that is, as though they consisted of negative electrons projected in straight lines with enormous velocity. We shall see further on that this velocity may be measured, and that the ratio between electric charge and mass has practically the same value as that found in the case of the cathode rays.

Subsequent researches have proved that radium and other strongly radio-active bodies also produce rays deviated somewhat less than the cathode rays by the electric and magnetic forces, but in the opposite direction. Hence it may be said that radio-active bodies emit three varieties of rays. It is 
probable that this is true of all bodies, since, even if some of these varieties of rays have not yet been found in the complex emission of certain radio-active bodies, this is in all probability due to their being less intense and hence less easy to detect. An example of this is found in Mme. Curie's polonium, which practically only emits rays whose deviation is opposite to that of the cathode rays.

The rays which are subject to deviation can only be considered as consisting in the emission of electrified particles. The direction of the deviation shows the sign of the charges of the particles, while the amount of the deviation permits an evaluation of the velocities with which the particles move, and in addition, of the ratio which exists between the charge and the mass of each; consequently the mass itself may be determined if we assume that its electric charge has that constant value which pertains to the electrolytic ion of hydrogen. We will now sum up the results of the research relating to radioactivity, adopting Rutherford's designation, 
$\alpha, \beta, \gamma$, for the three kinds of rays emitted by radium, and perhaps in general by every radio-active body.

The a-rays behave in a manner which confirms the hypothesis made by Strutt (36), which is that they consist of positive ions projected in all directions from the radio-active body. In fact, Rutherford (37) proved that they transport positive charges, while Becquerel (38) determined that their deviation in a magnetic field is in the opposite direction to that of the cathode rays.

If, for example, the rays emanate from a small quantity of one of the salts of radium contained in a little lead vessel, $P$ (Fig. I 2), they are propagated in the direction of the straight line $P C$; but if a magnetic field is set up perpendicular to the plane of the figure, the $a$-rays separate themselves from the others and curve in the direction of the arc $P A$.

It was found, on measuring the velocity and the ratio between the charge and the mass of the particles constituting the $a$-rays, that the velocity may attain a value about a 
tenth of the velocity of light, and that the ratio mentioned above would indicate that the particles possess a mass of atomic order. We may say, therefore, that the $a$-rays are identical with canal rays of high velocity.

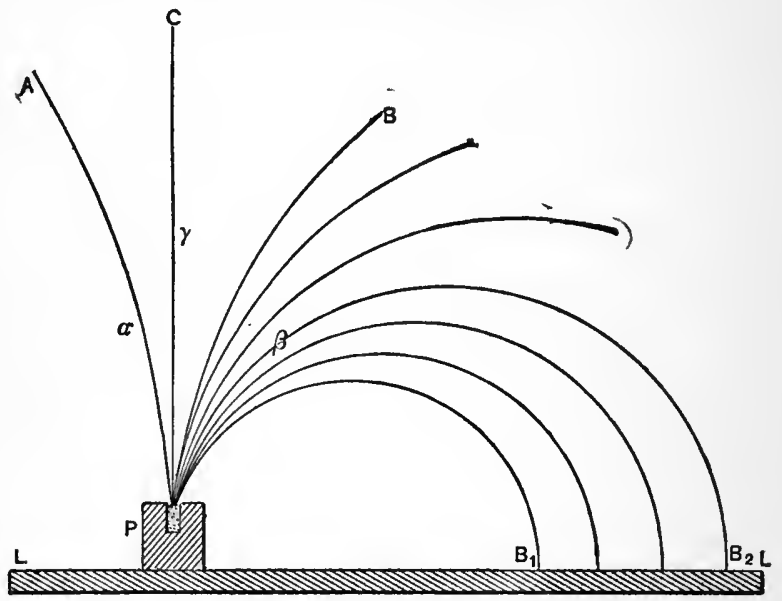

FIG. 12.

The ionization of a gas seems to be especially due to the $a$-rays, evidently as a result of collisions. The phenomenon is rather restricted if the gas which surrounds the radio-active body is at ordinary pressure. In fact, the $a$-rays from radium are only slightly penetrating, as either a layer of 
ordinary air ten centimeters thick, or a thin plate of aluminium less than a tenth of a millimeter thick, is sufficient to absorb the greater part of the rays.

The $\beta$-rays behave in all respects like very penetrating cathode rays. That is, they are negative electrons projected in all directions; their velocity is enormous, since it may attain a value but slightly inferior to the velocity of light. This result is deduced from the effect produced on the rays by the magnetic field, which was determined almost at the same time by Becquerel (39), Giesel (40), Meyer and Von Schweidler (4I), and Dorn (42). Instead of curving like the a-rays, they bend considerably more in the opposite direction, as is shown in Figure 12 . The curvature of some of the $\beta$-rays may be so great that they end by striking a photographic plate, $L L$, on which the lead vessel $P$ rests in the region $B_{1}, B_{2}$.

The production of rays curved to a greater or less degree by the magnetic field is due to the fact that the velocities of the various negative electrons, of which the $\beta$-rays are 
only the trajectory, have different values. The magnetic field causes those which move less rapidly to describe semicircles of small radius and those with the high velocity to describe the arcs of large radius. It is thus that the elongated image obtained on the photographic plate is explained. By means of this image it is easy to prove that the less deflected rays, consisting of electrons with great velocity, are also the more penetrating. In fact, a photographic plate placed in the path of the $\beta$-rays absorbs most strongly those rays which are the most deviated $\left(B_{1}\right.$, Fig. 12). Moreover, the variety of $\beta$-rays is quite great; thus, while some are stopped by aluminium foil one-hundredth of a millimeter thick, others are able to traverse several millimeters of lead. It was proved directly by the Curies (43) that the $\beta$-rays really transport negative charges. In their experiments the a-rays were arrested by a plate of aluminium so that only the effect of the $\beta$-rays was shown by the electrometer.

While the $a$ and $\beta$ rays are deviated in an electric or a magnetic field, the $\gamma$-rays are 
not affected at all. Thus those emitted by the radio-active body contained in $P$ (Fig. I2) preserve their rectilinear motion, $P C$, even when the magnetic field is set up.

The $\gamma$-rays, like the $\beta$-rays, are not homogeneous, there being a more penetrating and a less penetrating variety. This property renders them very similar to the Röntgen rays, and as such they are now generally considered. It is true it has been observed that the conductivity excited by the $\gamma$-rays in various gases is not proportional to that produced by the X-rays, and this seemed to establish a difference in the nature of the two sorts of rays. But recent experiment has shown that this dissimilarity in behaviour depends solely on the fact that the $\gamma$-rays in the aggregate are only comparable with the most penetrating $\mathrm{X}$-rays. In fact, the ratio between the conductivity produced in various gases by $\gamma$ and $\mathrm{X}$ rays tends toward unity, when a comparison is made between the former and Röntgen rays which are furnished by "hard" tubes and are made to pass through a lead plate before arriving at the gas to be ionized. 
When the three sorts of rays strike or traverse various bodies, they produce effects which differ according to the nature of the bodies themselves. This is particularly evident when one of the salts of radium is employed and certain of these effects have only thus far been observed with this substance. It is not possible to completely isolate the rays of one species from the others and to study separately the phenomena produced by them; but it is possible, by means of the interposition of absorbing plates to keep back the least penetrating rays, such as the $\alpha$, or the $a$ together with a part of the $\beta$ rays, or finally, to allow only the $\gamma$-rays to pass through; and this is sufficient in many cases to allow the effects produced by the one or the other to be recognized.

The effects produced by radio-active bodies, and in particular by radium, may be classified as luminous, chemical, electrical, mechanical, thermal, and physiological. Besides what has been previously stated, the following may be said with reference to these effects.

Phosphorescence and fluorescence seem to 
result especially from the action of the $a$ and the $\beta$ rays; but certain bodies become more brilliantly luminescent when they are struck by the $\alpha$-rays, others when they are struck by the $\beta$-rays. For example, hexagonal blende is particularly luminescent under the action of the $a$-rays from radium.

Crookes has constructed a small instrument called the spinthariscope (44), by means of which the effect produced by radium on a screen coated with phosphorescent sulphide of zinc is observed. A small particle of the radium salt is placed at a distance of about a half millimeter from the screen, which is viewed through a lens or microscope. Brilliant points appear here and there and immediately become extinguished and thus give an effect of scintillation. According to Crookes each luminous point is caused by the impact of a positive ion. According to Becquerel, the scintillation is due to the rupture or cleavage of the little crystals forming the phosphorescent films, since he obtained similar effects by crushing the body between two glass plates (45). In any case the singu- 
lar luminous phenomenon of the spinthariscope is only indirectly due to the supposed bombardment of the phosphorescent body by the a-rays.

The luminosity of the salts of radium has been attributed to a phosphorescence excited by the rays which they themselves generate. But it may be that the light is produced by the collision of the $a$ ions or of the $\beta$ electrons, not, in fact, with the molecules of the radio-active body, but with those of the surrounding gas or of the gases which are slowly released from the radium salt; and it might also be supposed that the cause of the luminous phenomenon is the transformation of the radium into an emanation, of which we shall speak later. However, it has been found by Sir William and Lady Huggins (46) that the spectrum of the light emitted by radium consists of lines coincident with some of those of nitrogen, and almost identical with that of the negative glow produced in air at ordinary pressure.

Recently Becquerel (47) has observed that the salts of uranium are also spontaneously 
luminous. In order to convince oneself of this, it is only necessary to look, at night, when the eye is well rested, at a small glass vessel containing uranium nitrate. The writer has, under such conditions, observed a very vivid scintillation when the vessel containing the salt was shaken; for this reason it is advisable not to shake the vessel too strongly if one wishes to verify the spontaneous emission of light and not confound it with that which is produced by concussions, and which is probably electrical in origin.

Phosphorescent substances are altered by the action of the rays emitted by radium and often change colour; glass becomes violet or blackish, and thus modified, it is thermoluminescent, since it becomes luminous when heated to about $500^{\circ} \mathrm{C}$. These modifications are probably chemical in nature, like those to which images on photographic plates are due.

Inasmuch as the rays from radium are unequally absorbed by bodies of different density, it is possible by their use to obtain radiographs similar to those, now so well 
known, which are obtained with X-rays. However, the latter are always very much the more perfect.

Radio-active bodies, and especially radium, produce still other chemical actions; thus according to Becquerel, a solution of bichloride of mercury and oxalic acid (6 $\frac{1}{2}$ grams of the bichloride, I $2 \frac{1}{2}$ of oxalic acid, and Ioo of water) deposits calomel under the action of the rays from radium; their action is therefore in this case similar to that of light rays. Under the action of the rays from radium ordinary phosphorus is transformed into red phosphorus, and oxygen into ozone. And it seems as though the changes in colour which are shown by glass, bariumplatino-cyanide, etc., should be attributed to chemical modifications. The last of these substances becomes at the same time less phosphorescent, but later reacquires this property when exposed to sunlight. It is a rather singular fact that the rays from radium do not sensibly modify the iodide of silver of a daguerreotype plate.

Since the $\alpha$ and $\beta$ rays consist in the 
emission of positive ions and negative electrons respectively, it is natural that they should produce an electric charge whenever the one variety and not the other is arrested. In this manner radio-active substances become a continuous source of electricity. Radio-active bodies should become electrified even when free motion is allowed to all the rays, if the quantity of positive electricity transported by the $a$-rays differs from the quantity of negative electricity carried away in the same time by the $\beta$-rays. Appropriate experiments have shown that this does not happen (48); and hence there must be a compensation between the charges of the two signs. If, however, the $\alpha$-rays are arrested by a sheathing of not too thin glass, this becomes positively electrified. This experiment was made by Wien with a small amount of bromide of radium placed in a glass tube wrapped in aluminium foil and hung by a wire in a vacuum tube. The gas must be removed from the region surrounding the small tube in order that the conductivity of the ionized gas may not cause 
the charge which collects to be dissipated. Under some conditions an electric spark

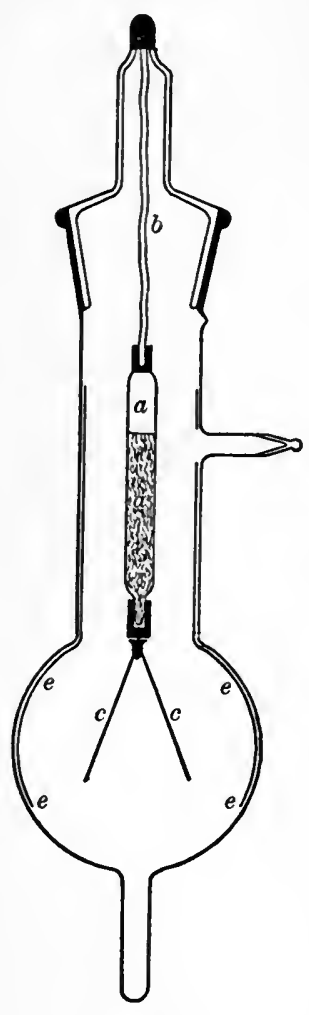

FIG. 13. may be obtained, as, for example, when a small tube containing radium (49) is opened.

An ingenious little piece of apparatus (Fig. I 3) which was devised by Strutt (50) demonstrates the continual production of electricity by radium. In a hermetically sealed glass vessel, in which a good vacuum has been formed, a small closed tube, $a$, is placed which contains a radio-active body. This tube, which is supported by an insulator of fused quartz, $b$, has its surface covered with a conducting film (for example, painted with phosphoric acid), and terminates in two gold leaves, $c c$, which form an electroscope. These leaves are always in motion. 
They spread apart little by little until they touch the strips of tin foil, $e e$, pasted on the walls of the vessel and communicating with the earth. Then they instantly fall together, only to slowly diverge again, and this goes on continuously. The effect is due to the positive charge left on the little tube by the $\alpha$-rays which are absorbed while the negative charge is carried away by the $\beta$-rays.

Another effect of an electrical nature, which is due to the rays from radium, is obtained, according to Curie (5I), in the following manner: conductors are placed in such a way that the discharge of an electric machine or an induction coil may follow two separate paths, in each of which an air gap is provided for the formation of the sparks. If these two air gaps are equivalent, so that the discharge takes place equally well in either of the two paths open to it, it is sufficient to bring the radium near one of the two air gaps in order that the spark may form at this one and not at the other. Therefore the rays from radium produce an action similar to that which, in certain circum- 
stances, is caused by the Röntgen rays, in that they tend to assist the formation of sparks.

Finally, another electrical effect of radium is the one which has been recently announced, namely, that a diminution in the electrical resistance of bismuth is produced when the radio-active substance is brought to within a very small distance of a thin plate of this metal.

Since the a-rays consist of positive ions, it follows that a body which emits them must necessarily send out matter as well; from a certain standpoint the same thing may be said of the $\beta$-rays, as we shall see later. It is to be expected, therefore, that there should be a continual diminution in the weight of radio-active substances. Heydweiller (52) thought that he had detected this; but Dorn (53) could not confirm this result in spite of the fact that he employed a body of greater radio-activity. Thus it would seem that we are dealing with a decrease in mass so small as to elude ordinary determinations.

In the case of radium, the emission of the 
various sorts of rays is accompanied by a continual production of heat. In fact, the Curies and Laborde (54) found that a small quantity of a radium salt continually maintained itself at a higher temperature than that of the surrounding air. The experiment, which is quite simple, is thus described by Mme. Curie. A closed glass flask containing seven decigrams of pure bromide of radium and a mercury thermometer are placed inside a double-walled glass vessel closed with a cotton stopper. A vacuum is produced between the two walls of the vessel, which is, in fact, one of those which are used to preserve liquid air because of the good heat insulation which may be obtained in this manner. A precisely similar thermometer, together with a similar glass flask, which, however, contains an inactive body such as chloride of barium, instead of the radio-active substance, are placed in a second glass vessel similar to the first. As soon as thermal equilibrium is established, it may be observed that the first thermometer registers a temperature about three degrees higher than the second. 
In order to measure the quantity of heat continuously evolved by the radium salt, a small quantity of it was introduced into a Bunsen calorimeter. From the quantity of ice which melted in a given time, it was deduced that the heat evolved by the radioactive body in one hour would be sufficient to melt a weight of ice equal to its own, or to heat an equal weight of water through about $80^{\circ} \mathrm{C}$. Thus we see that the quantity of heat furnished by radium is relatively great.

A thermal effect of another sort is that demonstrated by Georgiewski (55). According to this author, while the velocity with which a hot body cools does not vary when the gas which surrounds it is ionized by the rays from radium, the cooling itself is accelerated if the hot body is electrified, and especially if it is negatively electrified. This effect was observed not only with air, but also with other gases, and the result was the same whether all the rays from radium were allowed to act, or whether the a-rays were prevented from acting. 
When the rays from radium, and from radio-active bodies in general, strike an inactive body, they cause it to emit new rays which are called secondary rays. This emission is contemporaneous with the action of the incident rays, which distinguishes this phenomenon from that of induced radioactivity, of which we shall speak farther on. The property of exciting secondary rays pertains also to other radiations, such as X-rays and cathode rays, and in all cases the secondary rays differ from those which cause them. Thus it may be said that the X-rays are secondary rays produced by cathode rays, which may themselves be generated by X-rays (56). Moreover, these latter produce secondary rays of a nature similar to their own but which are less penetrating. Luminous and ultraviolet rays may produce cathode rays as secondary rays, etc. The rays from radioactive bodies produce effects of this sort, which, however, have not yet been studied in a thorough manner.

Besides the effects already described, the rays emitted by radium have a most pro- 
nounced action on living tissues and may even cause severe lesions hard to be cured. Becquerel and the Curies proved this to their cost (57). According to Danysz (58) a tube containing a strongly radio-active body placed on the skin of an animal caused a complete destruction of the epidermis and the dermis, though the action on the lower tissues was relatively quite weak. The nervous system is, on the contrary, quite sensitive to the action of radium, since lesions are soon formed which may result in paralysis and death.

The effect produced on bacteria is worthy of note; for, according to Aschkinass and Caspari (59), the development of certain species becomes arrested when they are subjected to these rays. The larvæ of some insects die, and the prolonged action of the rays from radium on the seeds of certain plants deprives them of the ability to germinate. It seems, moreover, that, in compensation for the deleterious actions which they exert, the rays emitted by radioactive substances may become useful in the 
cure of certain diseases, such as cancer and lupus.

The sensation of light which suffuses the field of vision when one of the radium preparations is brought close to the eye is not a direct physiological effect, but one which is explained by the phosphorescence of the parts which constitute the organ of sight. The sensation is produced even in the eye of a blind person, provided the retina and the optic nerve are in normal condition.

Finally, according to Bloch (6r), the action of the rays from radium on crystalline selenium is similar to that of light, since they increase its electrical conductivity.

In addition to the emission of $\alpha, \beta$, and $\gamma$ rays radio-active bodies, or at least radium and thorium, continually lose a part of their substance in another form. Certain experiments relative to the ionization of the air produced by thorium compounds led Rutherford to the discovery of the radio-active emanations. Before this Owens (62) had noticed a strange irregularity in the ionizing action of the rays from thorium oxide, but it was 
Rutherford who furnished the explanation of this $\left(6_{3}\right)$ by demonstrating that thorium continually emits radio-active particles, whose ionizing action reënforces that due to the $\alpha, \beta$, and $\gamma$ rays. Radium itself produces its own emanation, and the particles which form this emanation are distinct from those which constitute the $\alpha$ and $\beta$ rays, since the former diffuse slowly like the molecules of a gas while the latter are projected with enormous velocity.

In fact, the emanation mixes with the surrounding gas and may be carried with it from one place to another. Recent experiments show that the emanation behaves like a true gas, since, according to Traubenberg (64), not only the emanation contained in the city water of Freiburg (and, in general, in water from springs containing any radio-active substances whatsoever), but also the emanation from radium obeys the laws of Dalton and Henry, which hold for gases.

The emanation easily passes through narrow apertures or fissures through which an ordinary gas could only circulate with ex- 
treme slowness. It is only temporarily radioactive; that is, its radio-activity decays in a continuous manner to such an extent, that at the end of a minute the activity of the thorium emanation is reduced to one-half of the initial value, while that of radium loses its activity considerably less rapidly, being reduced to one-half only after about four days.

Rutherford and Soddy (65) have shown that the radium emanation condenses at a temperature of about $150^{\circ}$ below zero centigrade. In fact, a current of gas which has passed over a compound of radium and into a tube surrounded by liquid air, issues from the tube entirely robbed of the emanation, and the emanation itself returns to the gaseous state when the temperature is raised to the value mentioned above. It is easy to follow the motion of the emanation, because it is luminous and renders the glass tube which contains it luminous. The emanation from thorium condenses at a slightly higher temperature.

The emanation has the property of rendering bodies with which it comes in contact 
temporarily active. The radio-activity acquired in this manner by bodies which do not possess it on their own account was, it seems, first observed by the Curies, who called it induced radio-activity. According to Rutherford it is due to an invisible solid substance which the emanation deposits on bodies in unappreciable quantities, and which is soluble in certain acids but not in others. Upon evaporating the solution formed in this way, a radio-active residue is obtained. Moreover, it seems that the emanation adheres to the bodies which it has rendered active, and also, in a certain sense, is absorbed by them, since these bodies give off emanations of their own. Celluloid, india rubber, and paraffin absorb and then give up the emanation in appreciable quantities. Independently of this it has been found that a body which has become radio-active by induction, in a large measure loses this property when it is heated; this lost activity is acquired by surrounding cold bodies.

The presence of a negative charge on a body facilitates the production of induced 
radio-activity. It is even possible to cause a conductor which is strongly charged negatively to become radio-active by merely placing it for several hours in the open air, as has been shown by Elster and Geitel (67). According to Sella (68) the same result may be obtained in certain cases when the conductor is charged positively. These two experiments point to the existence in the atmosphere of an emanation analogous to those which are given off by radium and thorium.

Radio-activity may also be produced in substances which in themselves are inactive, by dissolving the inactive body in a liquid together with an active body and then separating the two by chemical methods. In this manner active bismuth is obtained after having been dissolved in a solution containing radium. Generally the body which has become radio-active in this way slowly loses its activity; and it is because of this that polonium has been considered by some to be bismuth which has been rendered active by solution. A salt of barium becomes radio- 
active, in a similar way, when it is derived from a solution of uranium, while this solution loses a part of its radio-activity, which it later slowly regains.

A phenomenon analogous to this, and one to which we shall return later, is that described by Rutherford, who was able to chemically separate thorium into two portions; the one which he called thorium-X is quite active and loses its radio-activity little by little as time goes on, while the other in time reacquires this lost property.

The continued evolution of heat and the emission of a-rays as well do not pertain exclusively to radium, but are also properties of the emanation which it evolves. If a radium salt is heated in a tube until so much of its emanation as it is possible to liberate is driven off and collected separately in a tube cooled by liquid air, then, when the tube containing the radium and that containing the emanation come into thermal equilibrium with the surrounding atmosphere, it is found that the quantity of heat produced in unit time by the radium diminishes, while that 
evolved by the emanation increases, in such a way that the sum of the two remains practically constant (69). If the emission of $a$ rays on the part of the radium and on that of the emanation are compared by means of the ionization which they produce, a behaviour exactly similar to that in the case of the emission of heat is noted. This leads one to suppose (70) that the heat evolved by the radium is due to the collision of the positive ions (a-rays) with the air molecules or with those of the radio-active substance itself. The velocity of the a-rays which has been found to attain a value of one-tenth of the velocity of light, is such as to satisfy this hypothesis; but at any rate the origin of the energy, of which radium seems to be an inexhaustible source, still remains unexplained.

It is probable that radio-activity is a property possessed to a greater or less degree by all bodies. In fact, recent experiments have shown that the speed with which a charged electroscope, for example that shown in Figure Io, becomes discharged, depends on the nature of the walls of the vessel in which it 
is inclosed $(7 \mathrm{I})$. This is attributed to the radio-activity of the walls themselves. It seems, moreover, that very penetrating radiations, which proceed perhaps from the atmosphere or from surrounding bodies, continually exist all about us and produce a slight ionization of gases, since the rapidity of discharge mentioned above becomes less, when the apparatus is surrounded by a thick shield of lead (72). Thus one might even suspect that the slight radio-activity of ordinary substances may be, at least in part, an emission of secondary rays excited by these radiations, which are supposed to be continually present.

The fact that air which has been drawn up from beneath the soil has an electrical conductivity greater than that of ordinary air is especially important. Moreover, it has been found that the water from many springs and wells, in particular the water supplied to the city of Cambridge (73), contains a radio-active gas which impregnates air made to bubble through the water and causes it to become feebly conducting. These two facts lead one 
to suppose with Elster and Geitel (74) that a small quantity of some radio-active substance, which may possibly be radium, exists in the ground, and that the emanation from this substance is carried by the ground air and is dissolved to a slight extent by spring water. Mr. Allen (75), who discovered that the water of the spring called "King's Bath" is radio-active, advances the idea, worthy of being taken into consideration in this connection, that the therapeutic action of mineral waters may be due at least in part to an emanation or to a radio-active gas contained in them; thus it would be easy to account for the fact, which is so often asserted, even if it has never been explicitly demonstrated, that mineral waters become less efficacious when exported.

The presence of radio-active emanations in springs which contain carbonic acid gas, and in certain thermal waters, as well as the relatively great radio-activity of the mud deposited from these waters, leads one to suppose that the proportion of radium, or of some other very radio-active substance, con- 
tained in the earth's crust, must increase with the depth below the surface (76). Hence it would seem very well worth while to institute an investigation of volcanic products.

Freshly fallen snow is radio-active. The writer has been able to ascertain that the conductivity of the air on a snowy day was more than double that on ordinary days.

In this chapter we have presented a long series of facts which have been, for the most part, ascertained with absolute certainty, but which at any time may assume a different aspect in the light of some future discovery. It would, therefore, be premature to formulate a theory of radio-activity at the present time, were it not that the nature of this phenomenon has been rendered so manifest by the properties which the radiations and emanations have been found to possess. Indeed, it is impossible to doubt that a radioactive body continually emits part of its own substance and that hence it should have a limited existence: the electron theory, in the form in which it will be presented in the last chapter, and according to which atoms are 
systems of electrons, seems designed expressly to confirm this belief. But a theory of radio-activity should also explain the origin of the energy which becomes available during the disintegration of radio-active bodies.

It is thought by some that this energy comes from an unknown radiation which is passing continually through all space. This radiation might impart enormous velocities to the constituents of the atoms of certain bodies - velocities great enough to separate these constituents from each other. Probably if such a radiation really did exist it would be absorbed by radio-active bodies. Thus it would seem that the radio-activity of a body might be reduced by surrounding it with other radio-active bodies. Probably no one has ever thought of experimenting in just this way, but Elster and Geitel (77) have found that the radio-activity of a body does not diminish when it is placed underground, for example in a mine, so that it is covered by a layer of the earth's crust at least eight hundred meters thick; and it is hardly prob- 
able that this should not absorb, at least to an appreciable degree, the radiations which are supposed to pass through space.

Hence it seems preferable to adopt the hypothesis that the origin of the energy of radio-active bodies is analogous to that of the thermal energy furnished by chemical combinations, with the difference that, while in the latter case we are dealing with the atoms which taken in the free state or issuing from other combinations unite together to form new molecules, in the case of the radio-active bodies we are dealing instead with electrons derived from unstable atoms, and which unite to form new and more enduring atoms. Certain experiments, and especially those of Rutherford and of his collaborators, render these atomic modifications and transformations highly probable and lead to the following consideration of the phenomena of radio-activity (78).

The atoms of the radio-active bodies are unstable systems of electrons. Every now and then some of these atoms subdivide into free negative electrons and groups of elec- 
trons with positive properties, or positive ions. The former constitute the $\beta$-rays; the latter the $\alpha$-rays. The emanation probably consists of these same positive ions or of modifications of them. If one portion only of the disintegrated atoms is radiated out into the surrounding space, the remaining portion will constitute a new body, which may be itself radio-active; and in this case its atoms will be subject to further division. The same thing may be said of the new atoms which constitute the emanation, as well as of the substance which this emanation deposits on inert bodies, thereby rendering them temporarily active (induced activity). The atomic transformations cease only when the electrons constitute stable atoms; that is, a non-radio-active substance.

For example, let us consider uranium. Crookes (79) and Becquerel (80) separated from this substance an active and a nonactive part. The former, which Crookes and Rutherford call uranium-X, in time loses its activity, while the latter slowly acquires it. This inactive uranium little by little becomes 
transformed into uranium-X, and the transformation is accompanied by radiation. At the same time the uranium-X becomes transformed into an unknown substance. A similar thing happens in the case of thorium, with this difference, however, that in addition to a transformation of inactive thorium into thorium-X accompanied by the emission of the usual rays, there is the production of an emanation as a result of a final disintegration of the atoms which constitute the thorium-X. The emanation itself is radio-active; that is, able to transform itself into other substances, among which is the substance which adheres to neighbouring bodies and causes induced radio-activity. Also in the case of thorium we are ignorant of what is the final and stable atomic condition.

The successive transformations of the radium atom are analogous to those of thorium, save that there apparently exists no radium-X. Thus there is a splitting up of the radium atoms and the formation of an emanation, or perhaps better of emanations, inasmuch as there seem to exist between the 
radium and the condensible emanation intermediate stages of transformation, which are the transformations taking place during the emission of the $\alpha, \beta$, and $\gamma$ rays. The emanation itself is radio-active, and in undergoing transformation gives rise to the substance producing induced radio-activity; that is, to a substance temporarily radioactive and thus in the stage of further transformation. Neither in the case of radium nor of the other radio-active bodies are we able to say what other successive aggregations are possible before a stable condition is attained; but the final non-radio-active substance has at least been found in the case of radium. It has in fact been recognized (8I) that in time the emanation of radium enclosed in a tube becomes modified; its spectrum slowly changes in aspect and ends by exhibiting the characteristic lines of helium. This gas, recently discovered in our atmosphere and in certain minerals, is one whose lines are found in the solar spectrum, and hence the name helium. The minerals in which it is found are, in general, those 
from which radium is obtained; a fact which is naturally explained by what has just been mentioned. ${ }^{1}$

A very recent experiment of the Curies and Dewar (82) seems to establish beyond all doubt the transformation of the radium emanation into helium. About four decigrams of pure and dry bromide of radium stood for three months in a small flask communicating with a Geissler tube. This was provided with two electrodes fused through the glass to allow the passage of an electric discharge, by means of which the gas contained in the tube could be rendered luminous. At first a good vacuum was produced in both flask and tube, and it was noted that the radio-active substance continuously evolved a gas which amounted to a cubic centimeter per month (under ordinary pressure). On passing a discharge through the

${ }^{1} \mathrm{E}$. Rutherford stated, in a paper read before the International Congress of Arts and Sciences in St. Louis (September 19-25, 1904), that the evidence at present indicates that the $\alpha$-particle is helium. See the important work by Rutherford entitled "Radio-activity," recently published in the Cambridge Physical Series.

- Translator. 
tube and on examining the spectrum of the light produced in this manner, the hydrogen and mercury lines were seen, the latter being due to the fact that a mercury pump was employed in obtaining the vacuum. Thus far no trace of helium had appeared.

The same bromide of radium was then placed in a quartz tube and heated until the salt fused. While the heating lasted, a pump was kept working in order to draw the gas, which was being given off, into a tube cooled by liquid air.

In this manner about 2.6 cubic centimeters of gas were collected, which was luminous on account of the emanation it contained. When this gas was brought into a Geissler tube, it showed only the spectrum of nitrogen; this was still the case after an attempt had been made to eliminate the nitrogen by means of condensation produced by cooling with liquid hydrogen. At this point the quartz tube containing the radium salt was hermetically sealed while it was being pumped out. Twenty days later, when the gas contained in the tube was rendered luminous by 
means of a discharge between external electrodes of tin-foil, the spectroscope clearly showed the entire spectrum of helium.

After this it seems difficult to doubt that helium is formed as a result of the radioactive transformations of radium. Nevertheless, many other proofs of this character will be necessary before the transformation of the chemical atoms may be considered as a demonstrated fact; that is to say, before the idea of the absolute invariability of the atoms, which with time has become so deeply rooted in our minds, is abandoned. 


\section{CHAPTER VI}

MASS, VELOCITY, AND ELECTRIC CHARGE OF THE IONS AND OF THE ELECTRONS

The time has now come to give some idea of the methods by which it has been possible to measure not only the ratio between the electric charge and the mass of the electrons or of the ions and the separate values of these two quantities, but also the velocity with which they move under various conditions. Such methods for the most part are based upon the effects which electric and magnetic fields, either acting separately or simultaneously, produce on the moving electrified particles; or they are based on the heat which is developed by the particles themselves when they strike an obstacle; or, finally, on the property which the particles possess of acting as nuclei for the condensation of vapours. Without entering into the details of the experiments and of the calcula- 
IIO MASS, VELOCITY, AND ELECTRIC CHARGE

tions which relate to them, let us examine these different phenomena.

First, let us consider the effect which the magnetic field produces on cathode rays, which move so as to render the wall of the discharge tube luminous at a point opposite the cathode, and whose direction is perpendicular to that of the field. For example, the tube in which the cathode rays are generated may be placed between the poles of a magnet. The electrons in motion, which constitute the cathode rays, deviate from their rectilinear path, and the rays themselves assume the form of circular arcs. In. fact, the magnetic field produces an electromagnetic force which affects the moving electron. This force is in the direction of that which the field would produce on an electric current coinciding with the trajectory, and hence it is perpendicular both to the trajectory and to the magnetic force; it has no effect on the speed of the electron, but only on the direction of its motion, which is circular and uniform. The electromagnetic force must then be equal and opposite 
MASS, VELOCITY, AND ELECTRIC CHARGE III

to the centrifugal force of this circular motion. Now the centrifugal force depends in a simple and known manner on the mass and the velocity of the particle and on the radius of its trajectory; and, on the other hand, the electromagnetic force is proportional to the charge of the electron and to its velocity, since the product of these two factors represents the intensity of the equivalent electric current. We thus arrive at a simple relation between the following quantities: first, charge and mass of the electron, or, more exactly, the ratio between the two; second, the velocity of the electron; third, the intensity of the magnetic field; fourth, the radius of the circular arc which is described. ${ }^{1}$ The last two quantities may be measured, and it is only necessary to know one of the others in order to calculate the remaining one.

If one assumes, as, in fact, at first it was assumed, that the velocity of the electron in

1 Let $e$ represent the charge of each particle, $m$ its mass, $V$ its velocity, $H$ the intensity of the magnetic field, $\rho$ the radius of the trajectory. The relation which exists between these quantities is $V=H \rho \frac{e}{m}$. 
II 2 MASS, VELOCITY, AND ELECTRIC CHARGE

the cathode rays is of the order of the magnitude of the molecular velocities in gases, one finds, for the ratio between charge and mass, a value of an order similar to that which holds for ions in electrolysis. Thus one would be led to suppose that the mass of the electron is of the order of magnitude of that of the material atom. Fortunately, it was recognized very soon that this assumption was incorrect, and, in addition to the magnetic deviation of the cathode rays, other effects were sought and other theoretical considerations were undertaken which might lead to a simultaneous determination of the velocity and of the ratio mentioned above. For example, it may be assumed that the velocity of the electron is such that its kinetic energy must equal the electric work done in the passage of the charge of the electron from the potential of the cathode to that of the anode. Such a method as this was adopted not long ago by Kaufmann (83) and Simon (84).

J. J. Thomson (85) determined the velocity of the electrons by measuring the negative 
MASS, VELOCITY, AND ELECTRIC CHARGE II 3

charge which they gave up on entering a hollow conductor connected with an electrometer, after having suffered a deviation in a magnetic field, and by measuring at the same time, by means of a thermo-electric couple, the energy transported by them. Thus two more relations may be added to the relation which exists between the quantities mentioned above. ${ }^{1}$ One of these states that the electric charge transported to the conductor (this is found by direct measurement) is equal to the number of electrons multiplied by the constant charge of each of them; the other states that the energy transported and transformed into heat by impact (and measured by means of the thermo-electric couple) is equal to the number of electrons multiplied by the kinetic energy of each, which is the half-product of the mass and the square of the velocity.

To the two unknown quantities which we had at first, we have thus added a new one,

${ }^{1}$ The two relations are $Q=N e, \frac{m V^{2}}{2} N=W$, where $Q$ is the total quantity of electricity transported, $N$ the number of electrons, and $W$ their kinetic energy. 
II4 MASS, VELOCITY, AND ELECTRIC CHARGE

which is the number of electrons set in motion during the experiment; but now we have three relations instead of the single one we had at first; hence by eliminating the number of electrons it becomes possible to calculate both the ratio between the charge and mass of each electron, and the velocity as well. ${ }^{1}$ The first results of any importance were obtained by this method. Values of the ratio between charge and mass of the electrons were obtained, which differed but slightly from each other when the rarefied gas in which the cathode rays were produced was changed (air, hydrogen, carbonic acid gas). In every case the value found clearly indicated that, if every electron represents an electric charge equal to that of an electrolytic ion, its mass must be, on the contrary, very much smaller than that of an ion of hydrogen. The values found for the velocity of the electrons showed, that it is very much

1 The elimination of $N$ from the three equations leads to the two following:-

$$
V=\frac{2 W}{Q H \rho}, \quad \frac{e}{m}=\frac{2 W}{Q H^{2} \rho^{2}},
$$

which serve to calculate $V$ and $\frac{e}{m}$. 
MASS, VELOCITY, AND ELECTRIC CHARGE II5

greater than the molecular velocities of gases, and that, in fact, it was in this instance about one-tenth of the velocity of light.

Another method for the determination of the velocity of the electrons of the cathode rays, which is based on the deviation produced by an electric field, is also due to Thomson (86).

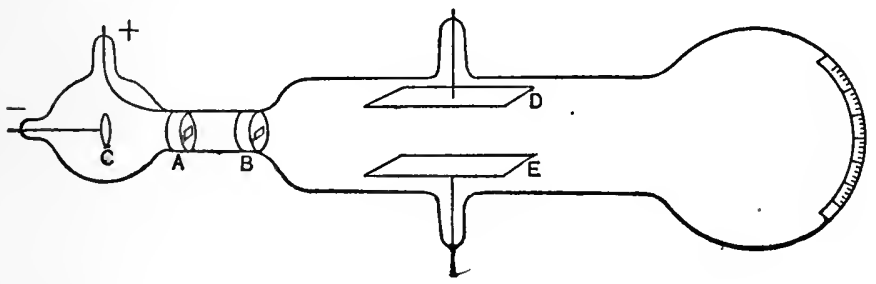

FIG. I4.

The cathode rays leave the cathode $C$ (Fig. 14) and are reduced to a narrow bundle by two thick metallic diaphragms $A$ and $B$, communicating with the earth and provided with narrow horizontal apertures, and then pass between two metal plates $D, E$. If these are oppositely charged, the rays should deviate from their rectilinear path, since the negative electrons, which consti- 
I16 MASS, VELOCITY, AND ELECTRIC CHARGE

tute the rays, will be attracted by the positive plate and repelled by the other. Hertz had been unable to obtain the expected deviation of cathode rays in an electric field, and neither did Thomson succeed in obtaining it at first, because the conductivity which a rarefied gas acquires when cathode rays pass through it makes it impossible to maintain the two plates at a sufficient difference of potential. But when the highest attainable rarefaction of the gas is reached, it is possible to obtain the effect and to see the displacement of the luminous spot at the end of the tube, due to the phosphorescence excited by the cathode rays. If, for example, the plate $E$ is positive and $D$ negative, the luminous spot descends, which shows that the path of the electrons has been curved downward.

In fact, the electric force, which may be assumed to be constant if the two plates are sufficiently large and near together, will cause each electron to describe a parabola, and thus the cathode rays will curve, precisely as a jet of water issuing from a horizontal tube curves under the influence of gravity. 
In the case of gravity the force is proportional to the mass of the moving body, and the acceleration is independent of the mass. Here, on the contrary, the force acting on an electron, and hence also the acceleration which it produces, is proportional to the electric charge; and since, for a given charge, and hence for a given electric force, the acceleration is in inverse ratio to the mass, it may be said that the acceleration itself, on which the form of the parabola depends in a known manner, is proportional to the ratio between the charge and the mass of the electron. Just as in the case of the magnetic deviation, so, also, in this case of the electric deviation there exists a relation which contains the above ratio and the initial velocity of the electron. Hence, if simultaneously with the electric field a magnetic field is produced, the lines of force of which are perpendicular both to the lines of electric force and the direction of the rays, it will be possible to calculate not only the ratio between the charge and mass of each electron, but also its velocity. Moreover, it 
is possible to arrange matters in such a way that the effects due to the two fields shall neutralize each other. Then the two quantities to be determined may be deduced from the intensity of each of the fields, and from the deviation produced when only one of them acts. ${ }^{1}$ In this manner Thomson obtained a value for the velocity about onetenth that of light, and a value for the ratio between the charge and mass of an electron in agreement with that found by the other method.

Similar measurements were made by $\mathrm{Mr}$. H. A. Wilson (87), who used a series of cathodes made of different metals and proved that the results obtained did not depend on the nature of the cathode.

Almost at the same time Thomson's method was used by Lenard (88), who ex-

${ }^{1}$ Denoting by $\theta$ the deviation produced separately either by the electric or by the magnetic field, by $l$ the length of the path along which the electron is exposed to the deflecting forces, by $F$ the intensity of the electric field, and by $H$ the magnetic field strength, we have

$$
V=\frac{F}{H} ; \quad \frac{e}{m}=\frac{F \theta}{H^{2} l} .
$$


MASS, VELOCITY, AND ELECTRIC CHARGE II9 perimented with the rays which bear his name; that is to say, with cathode rays which are made to issue through thin aluminium foil from the tube in which they are produced.

Lenard also (89) applied a new method, in which the electrons were subjected to the action of an electric field parallel to the

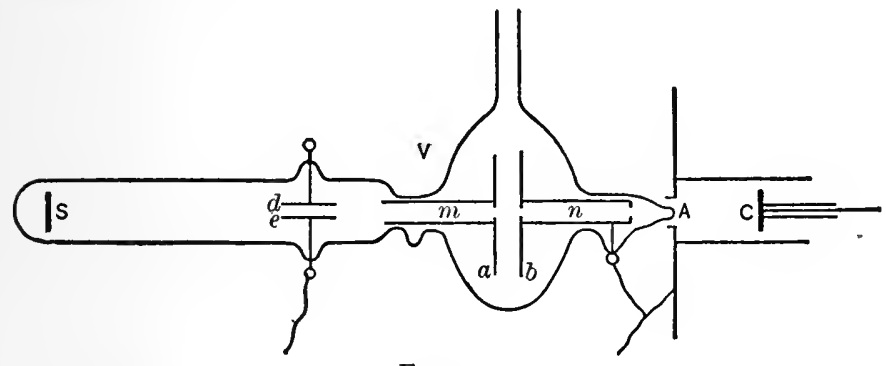

FIG. 15 .

direction of their motion. For this purpose the Lenard rays generated by the cathode $C$ (Fig. I 5) issue from a hole $A$, which is closed with aluminium foil, and thus penetrate into the apparatus $V$, containing a highly rarefied gas. In this apparatus a condenser is placed consisting of two parallel metallic disks, $a, b$, and having a small opening at the centre, through which the rays 
I20 MASS, VELOCITY, AND ELECTRIC CHARGE

coming from $a$ and directed toward the phosphorescent screen $S$, must pass. Two metallic tubes, $m, n$, protect the rays from the electric action of the disk $a$ which may be insulated and charged, while $b$ is kept always in communication with the earth. Hence it is only in the path between $a$ and $b$ that the electrons are exposed to an electric force which increases or diminishes their velocity according as the disk $a$ is positively or negatively charged. These same rays are, however, subjected farther on to a deflecting force, as in the preceding method, produced by a magnetic field or by a transverse electric field caused by the parallel disks $d, e$, and the deviation thus produced is measured.

As one might expect, for a given electric field between $d$ and $e$ the deviation depends upon the sign of the charge on $a$, since the velocity with which the electrons arrive in the field, producing the deviation, depends on the sign of this charge. By measuring this deviation and the intensity of the two fields the ratio so often referred to may be 
MASS, VELOCITY, AND ELECTRIC CHARGE I2I

calculated; the value which Lenard obtained by this method will be given farther on.

Finally, I will cite a most ingenious, but rather complicated, method which was adopted by Wiechert (90) in making a direct and accurate determination of the velocity of the cathode rays. By combining his results with those obtained by the method of magnetic deviation, he was able to calculate also the ratio between charge and mass of the electron.

But it is not only on the electrons of the cathode rays that measürements designed to determine their characteristic constants have been performed; other measurements have been made both on the negative electrons emitted by metals subjected to ultraviolet rays and on those emitted by incandescent or by radio-active bodies. Thus, J. J. Thomson (9I) resorted to an experimental method in which a magnetic field was caused to act upon the electrons emitted by an illuminated metal. By means of an almost identical experimental arrangement, the writer (92) had previously determined 
that a magnetic field reduces the transport of negative electricity from the body which receives the ultraviolet rays to neighbouring bodies; Thomson interpreted this fact in terms of the new theory, and availed himself of it in the following manner.

A small, negatively charged zinc disk $A B$ (Fig. I6), supported by a metallic rod $L$, is placed in a vessel containing highly rarefied air in such a way that it may be brought more or less close to a metal grating $C D$, which is parallel with the disk and connected with an

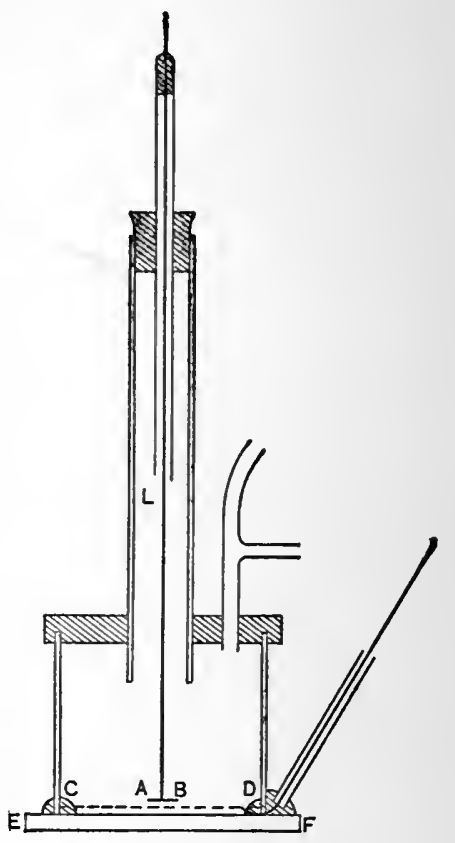

FIG. I6. electrometer. The vessel is closed by a quartz plate $E F$, in order that the more refrangible ultraviolet radiations caused by a shower of sparks passing between zinc wires 
may not be absorbed before they arrive at the charged disk. When the apparatus has been tested to make sure that the electrometer becomes discharged by the radiations, even when the disk $A B$ is relatively far from the grating, a magnetic field is produced the lines of force of which are parallel to the disk and to the grating. It is then found that if the distance between the disk and the

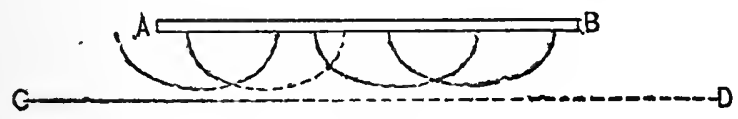

FIG. I7.

grating is increased to more than a certain amount, the transport of the negative electricity from $A B$ to $C D$ ceases almost entirely.

The mechanism to which the phenomenon is due is the following: before the magnetic field is set up the negative electrons which the radiations expel from the zinc disk $A B$ (Fig. I7) pass directly from $A B$ toward $C D$; but after a magnetic field perpendicular to the plane of the figure is produced, each electron describes a curve which may be shown to be a cycloid, and as a result the 
124 MASS, VELOCITY, AND ELECTRIC CHARGE electron after having gone a certain distance from the disk returns to it without having been able to reach the grating if the latter is too far distant. ${ }^{1}$ A few of the paths described by the electrons are shown as broken lines in the figure.

The maximum distance attained by an electron depends in a known manner upon the intensity of the electric field between the disk and the grating, upon the intensity of the magnetic field, and on the ratio be-

${ }^{1}$ Choosing the $X$-axis in the direction perpendicular to the disk and the grating, the $Y$-axis perpendicular to the $X$ axis and to the magnetic field, we have as the equations of motion :-

$$
\begin{aligned}
& m \frac{d^{2} x}{d t^{2}}=F e-H e \frac{d y}{d t} ; \\
& m \frac{d^{2} y}{d t^{2}}=H e \frac{d x}{d t} .
\end{aligned}
$$

Supposing the initial values of $x, y, \frac{d x}{d t}, \frac{d y}{d t}$, to be zero, the solution of these equations is :-

$$
\begin{aligned}
x=a(1-\cos b t), & y=a(b t-\sin b t), \text { in which } \\
a=\frac{F m}{H^{2} e}, & \text { and } b=H \frac{e}{m}
\end{aligned}
$$

The radius of the circle which generates the cycloid is $a$, and hence the maximum distance from the zinc disk which an electron may attain is : -

$$
2 \frac{F m}{H^{2} e} \text {. }
$$


MASS, VELOCITY, AND ELECTRIC CHARGE I25

tween the charge and mass of the electron. Hence it is possible to determine this ratio by measuring the first two quantities and the maximum distance between the disk and the grating beyond which the latter no longer receives a sensible charge. The accuracy of the determination is limited by the fact that the electrons do not all leave the disk with equal velocities, and hence the maximum distance from it which they attain is not the same for all.

Thomson (93) applied a similar method to the electrons emitted by an incandescent metal. Lenard (94) measured the ratio by resorting to the action of ultraviolet rays on metals in a vacuum; and finally Becquerel (95) and others determined the constants for electrons emitted by radio-active bodies. Without at present entering into the details of these last determinations and of others which have given analogous results, it will be well to tabulate the principal values of the ratio between the charge and mass of an electron in order that these values may be compared with each other. 


\section{MASS, VELOCITY, AND ELECTRIC CHARGE}

\begin{tabular}{|c|c|c|c|c|}
\hline $\begin{array}{l}\text { SOURCE OF } \\
\text { THE ELEC- } \\
\text { TRONS }\end{array}$ & EXPERIMENTER & Date & MEthod USED & $\begin{array}{c}\text { RATIO } \\
\text { BETWEEN } \\
\text { CHARGE AND } \\
\text { MASS }\end{array}$ \\
\hline $\begin{array}{c}\text { Cathode } \\
\text { Rays }\end{array}$ & J.J.Thomson & $\mathrm{r} 897$ & $\begin{array}{l}\text { Electric Deviation and } \\
\text { Magnetic Deviation. }\end{array}$ & $23^{1} \cdot 10^{15 *}$ \\
\hline $\begin{array}{c}\text { Cathode } \\
\text { Rays }\end{array}$ & J.J.Thomson & 1897 & $\begin{array}{l}\text { Magnetic Deviation, } \\
\text { charge transported, } \\
\text { and heat evolved. }\end{array}$ & $35^{1} \cdot 10^{15}$ \\
\hline $\begin{array}{c}\text { Cathode } \\
\text { Rays }\end{array}$ & Kaufmann & $\begin{array}{l}1897- \\
1898\end{array}$ & $\begin{array}{c}\text { Magnetic Deviation, } \\
\text { and } \\
\text { Potential Difference. }\end{array}$ & $55^{8} \cdot 10^{15}$ \\
\hline $\begin{array}{c}\text { Lenard } \\
\text { Rays }\end{array}$ & Lenard & 1898 & $\begin{array}{c}\text { Electric Deviation and } \\
\text { Magnetic Deviation. }\end{array}$ & $191.7 \cdot 10^{15}$ \\
\hline $\begin{array}{c}\text { Lenard } \\
\text { Rays }\end{array}$ & Lenard & I 898 & $\begin{array}{c}\text { Deviation and Electric } \\
\text { Field. }\end{array}$ & $204 \cdot 10^{15}$ \\
\hline $\begin{array}{c}\text { Cathode } \\
\text { Rays }\end{array}$ & Simon & 1899 & $\begin{array}{c}\text { Magnetic Deviation } \\
\text { and } \\
\text { Potential Difference. }\end{array}$ & $559.5 \cdot 10^{15}$ \\
\hline $\begin{array}{l}\text { Cathode } \\
\text { Rays }\end{array}$ & Weichert & 1899 & $\begin{array}{l}\text { Magnetic Deviation } \\
\text { and Velocity. }\end{array}$ & $\left\{\begin{array}{l}303 \cdot 10^{15} \\
465 \cdot 10^{15}\end{array}\right.$ \\
\hline $\begin{array}{l}\text { Ultra- } \\
\text { violet } \\
\text { Rays }\end{array}$ & J.J.Thomson & 1899 & $\begin{array}{l}\text { Diminution of the } \\
\text { discharge by the action } \\
\text { of a magnetic field. }\end{array}$ & $228 \cdot 10^{15}$ \\
\hline $\begin{array}{c}\text { Metal } \\
\text { heated to } \\
\text { Redness }\end{array}$ & J.J.Thomson & 1899 & $\begin{array}{l}\text { Diminution of the } \\
\text { discharge by the action } \\
\text { of a magnetic field. }\end{array}$ & $26 \mathrm{I} \cdot 10^{16}$ \\
\hline $\begin{array}{l}\text { Ultra- } \\
\text { violet }\end{array}$ & Lenard & 1900 & $\begin{array}{c}\text { Magnetic Deviation } \\
\text { and } \\
\text { Flectric Field }\end{array}$ & $345 \cdot 10^{15}$ \\
\hline $\begin{array}{c}\beta \text {-Rays } \\
\text { from } \\
\text { Radium }\end{array}$ & Becquerel & 1900 & $\begin{array}{c}\text { Electric Deviation } \\
\text { and } \\
\text { Magnetic Deviation. }\end{array}$ & $\begin{array}{l}\text { about } \\
300 \cdot 10^{15}\end{array}$ \\
\hline
\end{tabular}

* $23 \mathrm{r} \cdot \mathrm{ro}^{15}$ signifies the number obtained by writing 15 zeros to the right of the 23I; that is to say, the number $231,000,000,000,000,000$. The unit of charge adopted here is the electrostatic, which is defined as the quantity of electricity which repels a like charge placed at a distance of one centimeter with unit force; that is, with the force of one dyne. 
MASS, VELOCITY, AND ELECTRIC CHARGE 127

If account be taken of the great variety of phenomena in which the negative electrons make themselves manifest, and of the diversity of the methods adopted in the measurement of the ratio between the charge and the mass of each electron, the accord between the results is most remarkable. There can then remain no doubt as to the order of magnitude of this ratio since it turns out to be between 663 and r937 times greater than the analogous ratio for the hydrogen ion in electrolysis, which is equal to $0.289 \cdot 10^{15}$, and still greater than that which relates to the ions of other bodies. Therefore, the particles constituting the cathode rays and the $\beta$-rays from radio-active bodies cannot be atoms, but must be particles of very much smaller mass. Thus it is that the existence of masses which are much smaller than that of the smallest of the atoms of known substances, has been demonstrated in the surest possible manner and by purely physical methods.

The difference between the values found by various investigators for the ratio between 
I28 MASS, VELOCITY, AND ELECTRIC CHARGE

the charge and mass of the electrons is not solely due to errors in measurement, since the very accurate experiments made by Kaufmann (96) prove that this ratio varies with the velocity of the electrons, becoming rapidly smaller as the velocity approaches that of light. Kaufmann caused a magnetic and an electric field to act on the $\beta$-rays, emitted by one of the salts of radium, in such a way that the fields had a common direction perpendicular to that of the rays. The action of the magnetic force is to cause the rays to deviate in a certain direction, while that of the electric force is to cause the rays to deviate in a direction at right angles to this. Thus the experimental arrangement resembles the well-known one of the crossed prisms, in which two prisms cause light to undergo two successive deviations at right angles to each other. Just as in this latter experiment each coloured ray, separated from white light by the action of the first prism, suffers a new deviation due to the second prism, and these deviations may be separately measured, so also in Kaufmann's ex- 
MASS, VELOCITY, AND ELECTRIC CHARGE I29 periment the deviation of each of the $\beta$-rays, which differ as regards the velocity of the electrons which constitute them, may be measured.

By such a method as this Kaufmann found for the ratio between the charge and mass of the electrons values approximately equal to those obtained by other experimenters so long as he was dealing with electrons of relatively low velocity, or electrons constituting feebly penetrating $\beta$-rays, but he found much smaller values for the more penetrating rays; in fact, the ratio fell off to about one-half of the usual value for electrons whose velocity was about nine-tenths that of light.

Inasmuch as everything seems to point to the fact that the electric charge is always the same for all electrons, it must be held that their mass is not constant, but increases rapidly as their velocity approaches that of light. This result is of great importance, since it is in conformity with the supposition that the electrons do not possess a material mass in the ordinary sense of the word, but only an apparent mass due to the 
130 MASS, VELOCITY, AND ELECTRIC CHARGE

fact that they are electric charges in motion. But we shall return to this subject in the last chapter.

Wien (97) and Thomson (98) carried out measurements on the positive ions which constitute the canal rays, similar to those on the negative electrons. The first of these physicists obtained as a result a velocity of 3600 kilometers per second, and the value $0.009 \cdot 10^{15}$ for the ratio; Thomson found the ratio to be $0.012 \cdot 10^{15}$. Thus it is certain that the positive particles in the canal rays are not electrons, but are, instead, atoms or perhaps heavy electrified atomic groups.

The preceding conclusion relative to the small mass of the electrons is based on the hypothesis that the electric charge of the ions in gases is equal to that which is associated with every ion in electrolysis (or better, with every valency of the ion). From now on this may be considered an established fact rather than a hypothesis. In fact, the study of the diffusion of ions in gases (99) has led to the important conclusion that the electric charge of each ion is sensibly equal to that 
MASS, VELOCITY, AND ELECTRIC CHARGE I3I

which the ions have in electrolysis. But even before this result had been obtained Thomson (IOO) measured directly the charge of each ion in a gas, employing an extremely clever method of which we will now attempt to give an idea.

When air saturated with water vapour suddenly expands, a part of the vapour condenses in a cloud as a result of the cooling which accompanies the expansion, and each little globule generally has as a nucleus a particle of the microscopic dust which is ordinarily contained in the atmosphere. It seems, in fact, as though the presence of very minute bodies was required for the condensation of vapour, and that the condensation begins on the surface of these bodies whose radius of curvature must certainly be very small. At any rate, the presence of these corpuscles is favourable to the liquefaction of the vapour; for if the dust is carefully removed, it is necessary to expand the moist air considerably more in order that the cloud may form.

Mr. C. T. R. Wilson (IOI) showed that a gas containing ions behaves like air charged 
I32 MASS, VELOCITY, AND ELECTRIC CHARGE

with dust, since the ions themselves act as nuclei or centres of condensation for the vapour. And, in fact, an expansion of the air which may be too small to cause a cloud to form, may, on the contrary, be sufficient to precipitate the vapour when the air itself is ionized; for example, by passing Röntgen rays through it. That the change in behaviour of the gas is really due to the presence of ions was confirmed by the fact that the formation of the cloud is again retarded, or what amounts to the same thing, a greater expansion is necessary, when the ions are removed by the passage of an electric current through the gas.

The apparatus used by Thomson is somewhat complicated, but the essential part is a vessel which contains the moist air, and in which two horizontal conductors are placed one above the other. The upper, which may serve to close the vessel, generally consists of a disk of thin aluminium which is kept in electrical connection with the earth; the lower, which may consist of the mass of water which keeps the air above it saturated, is con- 
nected to an electrometer. At the proper moment the air is ionized by allowing X-rays or the rays emitted by a radio-active body to enter the vessel by passing through the aluminium plate. Ions may also be introduced into the vessel by charging the lower conductor negatively and by letting ultraviolet rays fall on its surface. In this case the aluminium disk is replaced by a metallic grating, the vessel is closed by a quartz plate, and a zinc or other metallic disk is chosen as the lower conductor.

In the first part of the experiment a measurement is made of the quantity of electricity which passes from one conductor to the other in unit time; that is to say, the intensity of the current which traverses the ionized air is measured. This is found by multiplying the diminution of potential in unit time, which is calculated from the decrease in the deviation of the electrometer, by the capacity of the system formed by the lower conductor and the electrometer. But, on the other hand, this current is due to the motion of the ions under the action of the electric field 
which exists between the two disks; and the intensity of the current may be calculated in terms of the following quantities: the strength of the electric field, the number of ions which exist in unit volume, the charge of each ion, and the velocity with which they move from one disk to the other. By equating the two values for the current strength a relation is obtained which contains the number of ions, their charge and their velocities, in addition to the numerical data resulting directly from the experiment. ${ }^{1}$ But the velocity of the ions in an electric field of known

${ }^{1}$ Calling the intensity of the field between the two disks $E$, the number of ions per cubic centimeter $N$, the velocity of the ions in a field of unit intensity $V$, the charge of each $e$, the area of the disks $A$, the intensity of the current from one disk to the other is obviously

\section{NVEeA;}

and if $C$ is the capacity of the insulated disk, $P$ the diminution of its potential in unit time, the equation which is mentioned in the text is

$$
N V E e A=C P \text {. }
$$

By measuring $E, A, C, P$, and by assuming for $V$ the value given by special experiments which Rutherford undertook to determine this velocity (480 centimeters per second in the case of ions produced in air by $\mathrm{X}$-rays), only $N$ and $e$ remain unknown. $N$ was measured in the manner described farther on in the text. 
intensity has been measured and is known; hence, in order to arrive at a determination of the charge of the ions, only the number which exist in unit volume remains to be measured.

The second part of the experiment serves to accomplish this. If a cloud is produced by a suitable expansion of the air, it is sufficient to count in some way the globules of water of which it is formed in order that the object of the experiment may be attained. In fact, assuming that each of the ions is a nucleus of one of the drops, the number of the former would equal the number of the latter. Now, to estimate the number of drops, it is sufficient to divide the total mass by the mass of each drop. The calculation of the total mass is based on the measurement of the lowest temperature which the air reaches in the act of expansion and on the measurement of the temperature of the air itself after the formation of the cloud $;^{1}$ the mass of each drop is inferred from its diameter, and

1 Calling the two temperatures $t, t^{\prime}$, the specific heat of the air at constant volume $C$, the latent heat of vaporization of 
I36 MASS, VELOCITY, AND ELECTRIC CHARGE

this in turn from the velocity with which it falls, or from the rate of descent of the upper surface of the cloud, which is always sufficiently sharp in outline. Naturally a relation exists between the dimensions of a sphere and the velocity with which it falls. While it is true that all bodies fall with equal velocity in a vacuum, this is not the case in air, the resistance of which more or less slows down their motion. In the case of a sphere this slowing down is the greater, the smaller the sphere, because, while the weight is proportional to the volume and hence to the cube of the diameter of the sphere, the resistance offered by the air is proportional to the surface, and hence to the square of the diameter. Therefore, as this is diminished, the weight falls off more rapidly than the resistance of the air. This is the reason why bodies reduced to fragments, such as powders and small drops, fall so slowly that they often seem to stand still.

the water $L$, the mass of the air in unit volume $M$, the mass of vapour condensed in a cubic centimeter of air $q$, we have

$$
L q=C M\left(t^{\prime}-t\right) \text {. }
$$


To determine the diameter of the drops of water, a formula ${ }^{1}$ is employed which contains this diameter in terms of the velocity of descent and the viscosity of the medium in which the particles move. The number of ions and therefore the charge of each may be calculated in the manner described above when the diameter is known.

The final result which Thomson obtained from a series of observations made on air ionized by $\mathrm{X}$-rays was that the charge of each ion is (in electrostatic units) $6.5 \cdot 10^{-\mathrm{x}}$, or 0.00000000065 . He deduced from his other experiments on the action of ultraviolet rays on zinc the value of $6.8 \cdot \mathrm{IO}^{-\mathrm{ro}}$, which differs only very slightly from the above result.

However, Thomson (IO2) himself later found that a correction should have been applied to these values. It has been proved that the condensation of vapour on the nega-

${ }^{1}$ If $V$ is the velocity with which a drop falls, $r$ its radius, $\mu$ the coefficient of viscosity of the air, and $g$ the acceleration of gravity, this formula is :-

For air $\mu=0.00018$.

$$
9 \mu V=2 g r^{2} .
$$


I 38 MASS, VELOCITY, AND ELECTRIC CHARGE

tive ions takes place with an expansion less than the minimum necessary in the case of condensation on positive ions, and that probably, in the experiment described, a few of the positive ions acted as nuclei. In fact, it is now known that, when the volume of the moist air is suddenly increased in the ratio of I to 1.25 , the negative and not the positive ions act as nuclei in the formation of the drops; it is only when the expansion is in the ratio of $I$ to $I .3$ I that the positive ions begin to take part in the phenomenon.

A series of new experiments, in which account was taken of this, led to the more accurate result of $3.4 \cdot 10^{-10}$ electrostatic units as the charge of each single ion.

Shortly after this, new research on the same subject was undertaken by Mr. H. A. Wilson (IO3), who adopted a slightly different method, which has the advantage of not requiring the evaluation of the number of ions existing in the air subjected to the Röntgen rays.

In this case also, by a sudden expansion of the air, the water vapour is condensed into 
MASS, VELOCITY, AND ELECTRIC CHARGE I 39

drops having an ion as a nucleus, but the cloud is made to form between two parallel metallic disks. When these are oppositely electrified, the velocity with which the drops fall is different from that which they possessed before the electric field was set up between the disks. Whether the change is an increase or decrease depends on the direction of the electric force. By measuring this velocity, sufficient data are available for the calculation of the charge of each drop. ${ }^{1}$

Without entering into further details relative to these very ingenious experiments, I will point out that they confirm the fact,

${ }^{1}$ Calling the velocity with which the drops fall when there is no electric field $v^{\prime}$, that when the field accelerates the fall $v^{\prime \prime}$, the intensity of the field $X$, the charge of the ion $e$, the mass of a drop $m$, the acceleration of gravity $g$, it is evident that we have

$$
m g v^{\prime \prime}=(m g+X e) v^{\prime} ;
$$

but from the equation

$$
9 \mu V=2 g r^{2}
$$

of the preceding footnote we may deduce

$$
m=3.14 \cdot 10^{-9} \cdot v^{\prime 3} \text {. }
$$

Hence, on eliminating $m$, we have

$$
e=3.14 \cdot 10^{-9} \frac{g}{X}\left(v^{\prime \prime}-v^{\prime}\right) \sqrt{\nabla^{\prime}},
$$

which permits a calculation of $e$ based on the measurement of $X, v^{\prime}$, and $v^{\prime \prime}$. 
I40 MASS, VELOCITY, AND ELECTRIC CHARGE

previously noted by Thomson, that when the intensity of the X-rays employed to ionize the air is very great, a number of ions may unite and form the nucleus of a single drop. In this case it follows that the charge of the drop is either twice, or three times, etc., that of a drop which has but a single ion as a nucleus. Wilson's experiments show that this really may happen, since the cloud separates into several horizontal layers which fall with different velocities. Evidently one of the layers is formed by drops having but one ion as a nucleus, another layer by those having two ions as a nucleus, etc. It is clear that, by taking this into account, a measurement of the velocity with which any one of the various layers falls may be used in calculating the quantity sought. The result which Wilson obtained $\left(3.1 \cdot 10^{-10}\right)$ differs but slightly from Thomson's result. This value is almost identical with that found by calculating the charge of the hydrogen ion in electrolysis, assuming the mass of the ion to have the value calculated from the kinetic theory of gases. 
MASS, VELOCITY, AND ELECTRIC CHARGE I4I

Thus we see that the numerical agreement which the theory would lead one to expect is substantiated within the limits of precision, which in this sort of research may at present be reasonably demanded. 


\section{CHAPTER VII}

THE ELECTRONS AND THE CONSTITUTION OF MATTER

ON account of the facility with which the electron theory lends itself to the formation of a model of the mechanism of physical phenomena, it possesses a decided utility, even in the minds of those who see in it only an instrument for research. In reality, the theory itself is hardly in its initial stages, and it would be premature to consider it just yet as a solid basis for a new system of natural philosophy. Nevertheless, since even from this point of view it is constantly acquiring importance, it will be useful to dedicate this last chapter to a concise exposition of the hypothesis, according to which matter is now coming to be considered as built up of electrons.

A rôle of fundamental importance is assigned to the electrons in this new mode 
of conceiving the constitution of bodies; but in order that it may be possible to explain the known phenomena in terms of the electrons, it is necessary to suppose them to be endowed with certain essential properties. Thus, for example, it is held that there exist electrons of two sorts, which are in a certain sense mutually antagonistic, namely, negative electrons and positive electrons; it is held that the first and not the second may exist in the free state; it is admitted that the separation of a negative electron takes place more easily, or with less expenditure of energy, from certain atoms, like those of the metals, than from others. However, the fundamental property attributed to the electrons is that, in their essence, they consist of electric charges acting on each other in the manner expressed by the formulæ of Hertz or Maxwell. It follows from this that the new theory does not pretend to give a reason for the cause of electric phenomena. This still remains a mystery. While formerly, starting with the existence of cosmic ether and that of ponderable matter, char- 


\section{I44 THE CONSTITUTION OF MATTER}

acterized by its principal attribute, inertia, the attempt was made to give a mechanical explanation for all phenomena; now, on the contrary, starting with the ether and the electrons, the attempt is made to construct, so to speak, ponderable matter out of these and to take account of the phenomena which it presents. Hence it may be said that the electron theory is much more a theory of matter than a theory of electricity; or, rather, in the new system electricity is set up in the place of matter, the existence of which was, on the whole, not much better understood than is the essence of the electrons at the present time.

In order to better understand the importance of the hypothesis and the fundamental attributes of the electrons, it will be well for us to consider synthetically the phenomena due to electrified bodies, either at rest or in motion. Following a line of reasoning due to Lodge (104), let us suppose that we bring into contact two bodies of different material and then separate them. They soon present that group of properties which constitute the 
two opposite electric states and, in particular, they attract each other and create the electric field which surrounds them. If one of the two bodies, say that which has the positive electric properties, is removed to an infinite distance, only the negative body need be taken into consideration. If we also suppose it to be very small, the electric field will be represented by rectilinear lines of force arriving at the body from all directions. The surrounding ether is now deformed, giving to this word the very broadest meaning; that is to say, it exists in a strained condition, which is shown not only by the tensions along the lines of force, which cause the apparent forces at a distance, but also by the transverse pressures. What the cause of this special state of the ether is, how it can be susceptible of a dual aspect, that is, how it can correspond to a positive and to a negative charge, are questions which we are absolutely unable to answer, just as we are unable to answer the question, what is the real structure and real nature of the entity which exists everywhere and which is called the ether. 
I46 THE CONSTITUTION OF MATTER

Let us now suppose that the small negatively electrified body moves with uniform speed; that is, we will suppose the special state of deformation just defined changes its position in the ether. It may be deduced from Maxwell's theory and from direct experiment as well, that this propagation of the ethereal strain from place to place produces the magnetic field. This may then be considered to be due to a deformation different in nature from the electric deformation but analogous to it, since tensions along the lines of force and pressures in the transverse direction also exist in the magnetic field. And if the motion is rectilinear, the lines of force are circles with their centres on the trajectory and lying in the planes perpendicular to this. A series of electrified bodies following each other with uniform motion has the properties of an electric current. Thus a constant current may be considered as a flow of equidistant electrons in uniform motion, and a variable current as a flow of electrons in variable motion, or of electrons which do not follow one another at equal intervals. 
If the small electrified body moves with non-uniform motion, the magnetic field which it creates is variable, and the phenomenon of induction takes place. If the motion is periodic, the phenomena of light occur. Every variation in the velocity of electrified bodies causes a variation in the magnetic field, this produces a variation in the electric field, and these two variations are propagated together with the velocity of light.

Let us now suppose that at a given moment we wish to increase the velocity of the electrified body, which we will suppose to move up to this moment with uniform speed. On account of the relations existing between the electric and magnetic force in an electromagnetic field, it is not possible to accelerate the motion of the electrified body without the expenditure of energy. In fact, an increase in the velocity results in a variation of the magnetic field, which in turn produces an electric force tending to oppose the acceleration of the motion. In the same way a decrease in the velocity is opposed by the generation of an electric force which tends 
to conserve the velocity of the electrified particle. In both cases the electromagnetic phenomenon is such as to simulate inertia, and the body by the mere fact of its being electrified behaves as though its mass were larger than it really is.

What has been said of the small electrified body holds for an electron, and its mass, which we have stated to be less than onethousandth that of the hydrogen atom, is at least in part not real, but apparent.

This species of apparent inertia, which an electrified body or an electron presents, is a manifestation of the phenomenon called selfinduction in the case of electric currents. In fact, if there are, instead of a single moving electron, a great number of such electrons following one another at small and equal intervals along the same path, these represent an electric current. An increase or diminution in the velocity of the electrons gives rise to an increase or diminution of the number which pass a given point on the path in unit time, and hence correspond to an increase or diminution in the intensity of the 
current. Now what has been said about the effect due to the variation in the velocity of a single moving electrified body or of a single electron, is substantially true of any number of electrons, and thus each variation in their velocity generates a force which tends to hinder the variation itself. Each change in the intensity of the current generates an electromotive force, which tends to oppose the change or to produce a new current in such a direction as to diminish the change. As is seen, this current is the extra current, and the electromotive force is the electromotive force of self-induction.

In summing up, it may be said that the electrons determine the production of the so-called electrostatic phenomena, when they are stationary; of the phenomena of magnetostatics and of constant currents when they are in uniform motion; of electromagnetic phenomena when they move non-uniformly, and of optical phenomena when they move with periodic motion. A sudden variation in the velocity of an electron which may be due, for example, to a collision, generates an 
I5O THE CONSTITUTION OF MATTER

electromagnetic wave in the ether analogous to the waves caused by an explosion in air. The X-rays are the manifestation of these waves.

We are now in a position to comprehend in what consists the modern hypothesis, according to which matter is built up of electrons. First of all, we may admit that the electrons are not matter, in the ordinary sense of the word; that is, they do not possess mass other than that which they seem to have by reason of their motion and electric charge. Kaufmann's experiment, referred to in the preceding chapter, renders this hypothesis very probable. He found, in fact, that the ratio between the charge and the mass of the moving electrons increases rapidly as the velocity approaches that of light. And since the hypothesis of a varying charge would be too improbable, it only remains to be supposed that the mass rapidly increases. Now such a result as this is in accord with the hypothesis according to which the mass of the electrons is entirely electromagnetic in its origin. 
Nothing prevents us from supposing that matter, and hence all known bodies, consist of aggregations or systems of electrons since the electrons, which may be considered as simple electric charges devoid of matter or as consisting in a modification of the ether symmetrically distributed about a point, perfectly simulate inertia by reason of the laws of the electromagnetic field and thus show the fundamental property of matter.

Therefore it may be admitted that a material atom is nothing but a system consisting of a certain number of positive and an equal number of negative electrons, and that the latter, or at least some of them, move about the remaining portion like satellites. Molecular and atomic forces would then be nothing but the manifestations of the electromagnetic forces of the electrons, and gravitation itself might be explained with these concepts as a basis. In fact, this has already been attempted.

If we suppose one or more negative electrons to be taken away from an atom, it becomes a positive ion, while the addition of 
one or more negative electrons to a neutral atom produces a negative ion. The manner in which various bodies behave when subjected to free electrons in motion, as in the case of the cathode rays, is such as to indorse the hypothesis. It has indeed been found that a body prevents the passage of the electrons, or absorbs the cathode rays, in proportion to its density; that is, in proportion to the total number of electrons, which constitute it, independently of the manner in which they are grouped to form chemical atoms of various kinds.

The electrons would seem to be, therefore, the elements of construction in the architecture of the atoms. When such a hypothesis as this is once adopted, the dogma of the invariability of the chemical atom or of the impossibility of transmutation of chemical substances is forever banished from science, since according to this hypothesis everything is built up of electrons. We have already seen how the phenomena of radio-activity seem to show transformations of this kind. If, in addition, it is held that all bodies are 
at least slightly radio-active and hence emit ions and electrons, these new views relative to the structure of matter become quite similar to those which were advanced as the basis of a general explanation of physical phenomena more than a half-century ago by a clever and original Italian physicist, Ambrogio Fusinieri (105). In spite of the fact that the concepts of this physicist have hitherto been open to objections and now have lost a part of their value in the light of subsequently discovered facts, one thinks at once of what he called attenuated matter emitted by all bodies, when one speaks of emanations sent out by radio-active bodies, or of electrons which, like a species of slow and invisible evaporation, are probably given off in a continuous manner by every material substance, and diffuse into surrounding space. 



\section{BIBLIOGRAPHY}

(I) W. Weber. Gesammelte Werke, Vol. IV, p. 279.

(2) A. Righi. Mem. della R. Acc. di Bologna, Series 5, Vol. VIII, p. 263 (1900).

(3) A. Righi. Rend. della R. Acc. di Bologna, February 18, 1894 .

(4) Any one who desires to gain a more thorough knowledge of the subject, which has only been treated in a very cursory manner in this chapter, would do well to consult -

J. Stark. Die Elektricität in Gasen. A. Barth, Leipzig, r902.

O. LODGE. Journal of the Institute of Electrical Engineers, 1903, Vol. 32.

J. J. Thomson. Conduction of Electricity through Gases. University Press, Cambridge, 1903.

(5) From an address by Sir William Crookes at the Chemical Congress held at Berlin in June, 1903.

(6) J. J. Thomson. Phil. Mag., Vol. 38, p. $35^{8}$ (1894).

(7) Q. Maiorana. N. Cimento, Series 4, Vol. VI, p. 336 (1897).

(8) J. Perrin. Comp. Rend., Vol. CXXI, p. ir 30 (I895).

(9) P. Lenard. Wied. Ann., Vol. 64, p. 279 (1898). (10) P. Lenard. Drude's Ann., Vol. 2, p. 359 (1900). 
(i I) J. J. Thomson and E. Rutherford. Phil. Mag., Vol. 42, p. 392 (1896).

(12) A. Righi. Mem. della R. Acc. di Bologna, Series 5, Vol. VI, p. 252 (I896).

(I3) A. Righi. Atti del R. Istit. Veneto, Series 6, Vol. VII (I889).

(I4) A. Righi. Rend. della R. Acc. dei Lincei, March 4,1888 .

(15) P. Lenard. Drude's Ann., Vol. I, p. 486 (Ig00).

(I6) A. RighI. Il moto dei ioni, etc. Attualità scientifiche I. Zanichelli, Bologna, 1903.

(17) Сh. Henry. Comp. Rend., Vol. CXXII, p. 312 (1896).

(i8) G. H. Niewenglowski. Comp. Rend., Vol. CXXII, p. 385 (1896).

(rg) H. Becquerel. Comp. Rend., Vol. CXXII, p. $420(1896)$.

(20) H. Becquerel. Comp. Rend., Vol. CXXII, p. 301 (1896).

(21) H. Becquerel. Comp. Rend., Vol. CXXII, p. 359 (1896).

(22) A. Righi. Rend. della R. Acc. di Bologna, February 9,1896 . This phenomenon was observed by various physicists at about the same time.

(23) G. C. Schmidt. Wied. Ann., Vol. 65, p. I4I (1898).

(24) S. Curie. Comp. Rend., Vol. CXXVI, p. I Ior (1897).

(25) P. ex S. Curie. Comp. Rend., Vol. CXXVII, p. 175 ( 1898$)$.

(26) P. et S. Curie et G. Bemont. Comp. Rend., Vol. CXXVII, p. I2r5 (r898). 
(27) A. Debierne. Comp. Rend., Vol. CXXIX, p. 593 (I899).

(28) J. Elster und H. Geitel. Wied. Ann., Vol. 69 (I899).

(29) F. Giesel. Ber. deutsch. chem. Ges., Vol. 33 , p. 3569 (Igor)

(30) K. Hofmann und E. Strauss. Ber. deutsch. chem. Ges., Vol. 33, p. 3126 (1900); Vol. 34, p. $3 \circ 35$ (I901); Vol. 35, p. I 453 (I9०2).

(3I) K. Hofmann und V. Wölfl. 'Chem. Ber., Vol. 36, p. 1040 (1903).

(32) W. Marckwald. Phys. Zeitschr., Vol. IV, p. 5I (1902).

(33) F. Giesel. Chem. Ber., Vol. 36, p. 728 (r9o3).

(34) C. Baskerville. J. Amer. Chem. Soc., Vol. 23, p. 761 .

(35) R. Blondlot. Comp. Rend., Vol. CXXXVI, p. 735 (1903), and succeeding numbers.

(36) R. J. Strutt. Phil. Trans., Vol. 196, p. 525 (I90I).

(37) E. Rutherford. Phil. Mag., May, 1903, p. 177. (38) H. Becquerel. Comp. Rend., Vol. CXXXVI, p. 43 r (1903).

(39) H. Becquerel. Comp. Rend., Vol. CXXIX, p. 9 I2 (I899).

(40) F. Giesel. Wied. Ann., Vol. 69, p. 834 (r899).

(4I) S. Meyer und E. von Schweidler. Phys. Zeitschr., Vol. I, pp. 90, I 33 (1899).

(42) E. Dorn. Comp. Rend., Vol. CXXX, p. I 126 (1900).

(43) P. et S. Curie. Comp. Rend., Vol. CXXX, p. 647 (1900). 
(44) W. Crookes. Proc. Roy. Soc., Vol. LXXI, p. 405 (1903).

(45) H. Becquerel. Comp. Rend., October 27, 1903, p. 629.

(46) W. Huggins and Lady Huggins. Proc. Roy. Soc., p. 196 (1903).

(47) H. BecQuerel. Comp. Rend., January 25 , I904.

(48) W. Wien. Phys. Zeitschr., Vol. IV, p. 624 (1903).

(49) E. Dorn. Phys. Zeitschr., Vol. IV, p. 507 (I903).

(50) R. J. Strutr. Phil. Mag., November, r903, p. 588.

(51) P. CuRIE. L'Electricien, January 23, 1904.

(52) A. Heydweiller. Phys. Zeitschr., Vol. IV, p. 8r (I902).

(53) E. Dorn. Phys. Zeitschr., Vol. IV, p. 530 (1902).

(54) P. Curie et A. Laborde. Comp. Rend., Vol. CXXXVI, p. 673 (1903).

(55) N. Georgiewski, Jour. de la Soc. Phys. Chim. russe, Vol. 35, p. 353 (1903).

(56) A. Righi. Mem. della R. Acc. di Bologna, Series 5, Vol. X, p. 595 (1903).

(57) H. Becquerel et P. Curie. Comp. Rend., Vol. CXXXII, p. I289 (rgor).

(58) J. Danysz. Comp. Rend., Vol. CXXXVI, p. 46r (1903).

(59) Aschkinass und Caspari. Arch. für die Ges. Physiol., Vol. 86 (IgOI).

(60) Danlos. Soc. de derm., November 7, rgor. Hallopau et Gadaud. Soc. de derm., July 3, 1902. 
(6r) E. Bloch. Comp. Rend., Vol. CXXXII, p. 9I4 (I90I).

(62) R. B. Owens. Phil. Mag., Vol. 48, p. 36I (1899).

(63) E. Rutherford. Phil. Mag., Vol. 49, pp. I, I6I (1900).

(64) H. R. v. Traubenberg. Phys. Zeitschr., March I, 1904, p. I30.

(65) E. Rutherford and F. Soddy. Phil. Mag., May, 1903, p. 56I.

(66) P. et S. Curie. Comp. Rend., Vol. CXXIX (1899).

(67) Elster und Geitel. Phys. Zeitschr., Vol. III, p. 76 (I9OI).

(68) A. Sella. Il Nuovo Cimento, Vol. 3, p. 138 ; Vol. 4, p. I3I (1902).

(69) E. Rutherford and H. T. Barnes. Phil. Mag., February, I904, p. 202.

(70) O. Lodge. Nature, April 2, I903.

(7 I) J. J. Thomson. Nature, February 26, 1903.

R. J. Strutt. Phil. Mag., June, 1903, p. 680.

J. C. Maclennan and E. F. Burton. Phil. Mag., June, 1903, p. 69I.

(72) E. Rutherford. Nature, r903, p. 5 II.

H. Lester Cooke. Phil. Mag., October, 1903, p. $4 \circ 3$.

(73) J. J. Thomson. Nature, I903, p. 90.

(74) J. Elster und H. Geitel. Phys. Zeitschr., I904, p. II.

(75) H. S. Allen. Nature, August I3, 1903.

(76) See note (72).

(77) J. Elster Und H. Geitel. Beiblätter, 1899, p. 443 . 
(78) E. Rutherford and F. Soddy. Phil. Mag., May, 1903 , p. 576.

(79) W. Crookes. Proc. Roy. Soc., 1900, Vol. 66, p. 419 .

(80) H. Becquerel. Comp. Rend., Vol. CXXXVI, p. 137 (1900).

(8i) E. Rutherford and F. Soddy. Phil. Mag., April, 1903, p. 44I.

W. Ramsay, Nature, August, 1903, p. 354 .

W. Ramsay and F. Soddy. Phys. Zeitschr., September 18,1903, p. $65 \mathrm{I}$.

(82) Dewar et Curie. Comp. Rend., January 25, I904, p. I9O.

(83) W. Kaufmann. Wied. Ann., Vol. 6r, p. 544; Vol. 62, p. 596; Vol. 65, p. 43 (1898).

(84) S. Simon. Wied. Ann., Vol. 69, p. 589 (1899).

(85) J. J. Thomson. Phil. Mag., Vol. 44, p. 293 (1897).

(86) J. J. Thomson. Phil. Mag., Vol. 44, p. 293 (1897).

(87) H. A. Wilson. Proc. Camb. Phil. Soc., r9or, p. 179.

(88) P. Lenard. Wied. Ann., Vol. 64, p. 279 (1898).

(89) P. Lenard. Wied. Ann., Vol. 65, p. $5 \circ 4$ (1898).

(90) E. Wiechert. Wied. Ann., Vol. 69, p. 739 (1899).

(9 I) J. J. Thomson. Phil. Mag., Vol. 48, p. 547 (1899).

(92) A. Righi. Mem. della R. Acc. di Bologna, Series 4, Vol. X, p. 110 (1890).

(93) J. J. Thomson. Phil. Mag., Vol. 48, p. 547 (x899).

(94) P. Lenard. Drude's Ann., Vol. 2, p. 359 (x900).

(95) H. Becquerel. Rapports du Congrès de Physique de Paris, Vol. III, p. 47. 
(96) W. Kaufmann. Gött. Nach., November 8, I901 ;

July 26, I902; March 7, 1903.

(97) W. Wies. Wied. Ann., Vol. 65 , p. 440 (1898).

(98) J. J. Thomson. See note (4), p. I I9.

(99) J. S. Townsend. Phil. Trans., Igoo, p. 259.

(100) J. J. Thomson. Phil. Mag., December, I8g8, p. 528 .

(Iоr) C. T. R. Wilson. Phil. Trans., r897, p. 265.

(זо2) J. J. Thomson. Phil. Mag., March, 1903, p. 346.

(ıо3) H. A. Wilson. Phil. Mag., April, 1903, p. 429.

(I04) O. Lodge. See note (4).

(105) A. Fusinieri. Memorie, Padova, 1844, 1846, 1847 . 



\section{INDEX}

[THE NUMBERS REFER TO THE PAGES.]

a-rays, 73 .

Absorption of, 74 .

Magnetic deviation of, 73 .

Nature of, 73 .

Velocity of, 74, 97 .

Actinium, 63 .

Anode rays, 52 .

Aschkinass and Caspari :

Effect of radium on bacteria, 90 .

$\beta$-rays, 75 .

Absorption of, 76 .

Magnetic deviation of, 75 .

Negative charge transported by, 76.

Baskerville:

Carolinium, 65.

Becquerel:

Magnetic deviation of a-rays, 73 .

Magnetic deviation of $\beta$-rays, 75 .

Rays, 58, 6r.

Theory of spinthariscope, 79 .

Bemont :

Preparation of radium, 62.

Canal rays, $\mathbf{5 2}^{2}$.

Carolinium, 65.

Cathode:

Dark space, 3 r.

Cathode rays, $3^{2}$.

Ionization by, 48 .

Transport of charge by, 35 .

Velocity of, 35.

Charge of electron:

Ratio to mass, II8, I2I, 125.

Variation of ratio, 128.
Charge of ion, I3I et seq.

Curie, M.:

Discharging action of radium rays, 85 .

Curie, Mme.:

Preparation of radium, 66.

Radio-activity of thorium, $6 \mathrm{r}$.

Curie, M. and Mme.:

Charge transported by $\beta$-rays, 76 . Preparation of polonium, 62 .

Curie, M. and Mme. and Laborde: Evolution of heat by radium, 87 .

Crookes, Sir W. :

Radiant matter, 34 .

Spinthariscope, 79 .

Danysz :

Physiological effects of radium, go.

Debierne:

Preparation of actinium, 63 .

Dewar and Curie:

Production of helium, ro6 et seq.

Dissociation :

Electric, 9.

Electrolytic, Io.

Dorn :

Loss of weight of radium, 86.

Magnetic deviation of $\beta$-rays, 75 .

$\frac{e}{m}$, I18, I21, 125, 130 .

Variation of, 128, 130.

Electric field :

Deviation of rays by, II $5 \mathrm{ct} \mathrm{seq.}$

Electric shadow, 49. 
Electrolysis, 3.

Electron :

Charge of, 26, 129.

Mass of, 37, 129, 150 .

Elster and Geitel :

Excited activity in atmosphere, 95 .

Faraday :

Dark space, 30.

Fourth state of matter, 33 .

Fluorescence, 32.

$\boldsymbol{\gamma}$-rays, 77.

Georgiewski :

Thermal effect, 88.

Giesel :

Magnetic deviation of $\beta$-rays, 75 .

Radio-lead, 63.

Helium :

Produced from radium emanation, 106 et seq.

Heydweiller :

Loss of weight of radium, 86.

Hofmann and Strauss :

Radio-lead, 63.

Hofmann and Wölf :

Radio-lead, 64.

Huggins, Sir W. and Lady:

Spectrum of radium, 80 .

\section{Ions:}

As nuclei of condensation, I32. Charge, mass, and velocity of, I30, 137 et seq.

Ionization, 40.

By impact, 45, 50 .

Causes of, 45 .

Due to Lenard rays, 47.

Kaufmann:

Variation of $\frac{e}{m}, 128,150$.

Lenard:

$$
\frac{e}{m}, 118 \text { et seq. }
$$

Transport of charge by cathode rays, 35.

Lenard rays :

Ionization by, 47 .

Lorenz :

Electromagnetic theory of light, I3.

Magnetic field:

Effect on a-rays, 73 .

Effect on $\beta$-rays, 75 .

Effect on cathode rays, 1 IIO.

Effect on circular vibration, 20 et seq.

Maiorana:

Velocity of cathode rays, 35 .

Marckwald:

Radio-tellurium, 64.

Meyer and Von Schweidler:

Magnetic deviation of $\beta$-rays, 75 .

Perrin :

Transport of charge by cathode rays, 35 .

Pitchblende, 62.

Polonium, 62.

Rays emitted by, 72.

Radiant matter, 34 .

Radiation:

From radio-active bodies, 69 .

Radio-active bodies:

Chemical actions due to, 82 .

Effects produced by, 78 .

Evolution of heat by, 96 .

Loss of weight by, 86 .

Radio-active emanations, gr.

Gaseous nature of, 92.

Presence in the atmosphere, 95.

Radio-activity :

Induced, 65, 94.

Of snow, roo.

Of spring water, 98 .

Radio-lead, 63.

Radio-tellurium, 64. 
Radium, 63.

Evolution of heat by, 87 .

Physiological effect of rays from, go.

Preparation of, 65 et seq.

Spectrum of, 68,80 .

Rutherford :

Induced radio-activity, 94 .

Radio-active emanations, 9I et seq.

Thorium-X, 96 .

Transport of charge by a-rays, 73 .

Rutherford and Soddy:

Theory of atomic disintegration, I02 et seq.

Saturation current, 43.

Schmidt :

Radio-activity of thorium, $6 \pi$.

Secondary rays, 85 .

Selenium :

Effect of rays from radium on, 91 . Sella :

Excited activity in the atmosphere, 95 .

Spinthariscope, 79.

Strutt :

Nature of a-rays, 73.

Production of electricity by radium, 84 .
Theory of atomic disintegration, ro2 et seq.

Thomson, J. J.

Charge of the ion, I3I.

Velocity of cathode rays, 35 .

Velocity of the electrons, II2 et seq.

Velocity and $\frac{e}{m}$ of the positive ions, 130 .

Thorium, 6r.

Thorium-X, 96, 104 .

Uranium :

Radio-active compounds of, 57 , 6r.

Uranium-X, Io3.

Weber :

Atomic theory of electricity, 5 .

Wien :

Velocity and $\frac{e}{m}$ of positive ions, rзo.

Wilson, C. T. R.

Ions as nuclei of condensation, I32.

Wilson, H. A.

Charge of the ion, 138 .

Zeeman effect, $I_{4}$ et seg. 



\section{Principles of Inorganic Chemistry}

BY HARRY C. JONES, Ph.D.

Professor of Physical Chemistry in the Johns Hopkins University

Cloth

8vo

$\$ 4.00$ net

"I regard the book the most important contribution to didactic chemistry made in this country in recent years. I shall have frequent occasion to use the work in my classes."

\section{Professor James Lewis Howe, Department of Chemistry, Washington and Lee University, Lexington, Va.}

"I consider it a most lucid and concise treatment of the subject, which will make the work a most admirable text-book."

$$
\begin{aligned}
& \text { William L. Dudley, Professor of Chemistry, } \\
& \text { Vanderbilt University, Nashville, Tenn. }
\end{aligned}
$$

"The student whose training in general chemistry has lacked illumination by modern views, whether on account of the ignorance, conservatism, or deliberate preference of his first instructor, will find in this book abundant opportunity for bringing his conceptions up to date and getting in touch with the science as it is. To such a student the book will be most suggestive and stimulating. Dr. Jones is to be congratulated on having produced one of the best of the recent attempts to apply physico-chemical conceptions to undergraduate instruction."

Alexander Smith,

In Fournal of American Chemical Society, Easton, $\mathrm{Pa}$.

\section{THE MACMILLAN COMPANY}




\title{
Elements of Inorganic Chemistry
}

\author{
By HARRY C. JONES, Ph.D. \\ Professor of Physical Chemistry in the Johns Hopkins University
}

\section{Cloth}

\section{$\$ 1.25$ net}

\footnotetext{
"I have examined the book, and am delighted with the interesting and up-to-date presentation of the elementary facts and generalizations of Inorganic Chemistry."

William A. Blanchard, Department of Chemistry, De Pauw University, Greencastle, Ind.
}

"The appearance of this book inaugurates a new epoch in the teaching of chemistry to beginners. Dr. Jones's application of modern electrolytic laws will do an immense amount of service, not only to chemistry, but to physiology as well."

R. C. Schiedt,

Professor of Physiology and Chemistry, Franklin and Marshall College, Lancaster, $\mathrm{Pa}$.

"It differs from the ordinary ' elementary' book in the obvious lack of unessential details, which often needlessly take up space in books of this kind. I was also pleased with the clear statement of the theoretical and physical-chemistry facts. These are simply enough expressed to be readily understood by the beginner, which is often not the case."

Dr. Herbert R. Moody, Professor of Chemistry, Hobart College, Geneva, N.Y.

\section{THE MACMILLAN COMPANY}




\title{
THE \\ Elements of Physical Chemistry
}

\author{
By HARRY C. JONES, Ph.D.
}

Professor of Physical Chemistry in the Johns Hopkins University

Cloth 8vo \$4.00 net

\section{COMMENTS}

"I have devoted considerable time to the perusal of the book, and am genuinely pleased with it. It shows most conscientious work on the part of the author; and in discussing the most recent research, in so far as it is of fundamental interest, the author has given the book a value far beyond that of the usual elementary treatise."

StEwarT W. Young,

Associate Professor of Chemistry, Stanford University.

"Professor Jones has presented the principles of the subject with the ardor of an investigator in that line of work. The facts are presented with great clearness, and the book can hardly fail to interest every one who would keep pace with the investigations in physical chemistry."

LeVERETT MEARs, Professor of Chemistry, Williams College.

"It seems to me a very timely and valuable work. I am glad to have it for my own use, and shall certainly recommend it to my associates and students."

W. O. Atwater, Professor of Chemistry, Wesleyan University.

"This scholarly volume should have a wide and long-continued sale. It impresses me as an exceedingly clear and well-balanced treatment."

Henry Crew,

Professor of Physics, Northwestern University.

\section{THE MACMILLAN COMPANY}




\title{
The Theory of Electrolytic Dissociation .
}

\section{AND SOME OF ITS APPLICATIONS}

\author{
BY HARRY C. JONES, Ph.D.
}

Professor of Physical Chemistry in the Johns Hopkins University

Cloth $12 \mathrm{mo} \quad \$ 1.60$ net

"I have tried several of the German works, small and large, and I have no hesitation in saying that Professor Jones's presentation is the simplest, clearest, and most adequate in any language."

\section{Professor S. F. BARKer, Johns Hopkins University.}

"It is a text-book which will supply a long-felt want to teachers who have to do with students unfamiliar with the German language. . . . The examples chosen are apt, well described, and clearly explained. With this book and Professor Walker's Introduction to Physical Chemistry our students have now a remarkably good presentation of the subject in English."

\section{Professor J. W. WALKER, McGill University.}

"The aim of this little work is to present in a connected form the investigations which lead up to and upon which the electrolytic dissociation of Arrhenius is based. The book will be welcome to a large class of readers who desire some knowledge of the more recent developments in physical chemistry, but who have not the time or opportunity for consulting the original literature of the subject."

The Technology Review.

"The book will undoubtedly be useful to those who wish to gain a clear notion of some of the chief points of modern physical chemistry."

American Fournal of Science.

\section{THE MACMILLAN COMPANY}






$$
\text { 、 }
$$





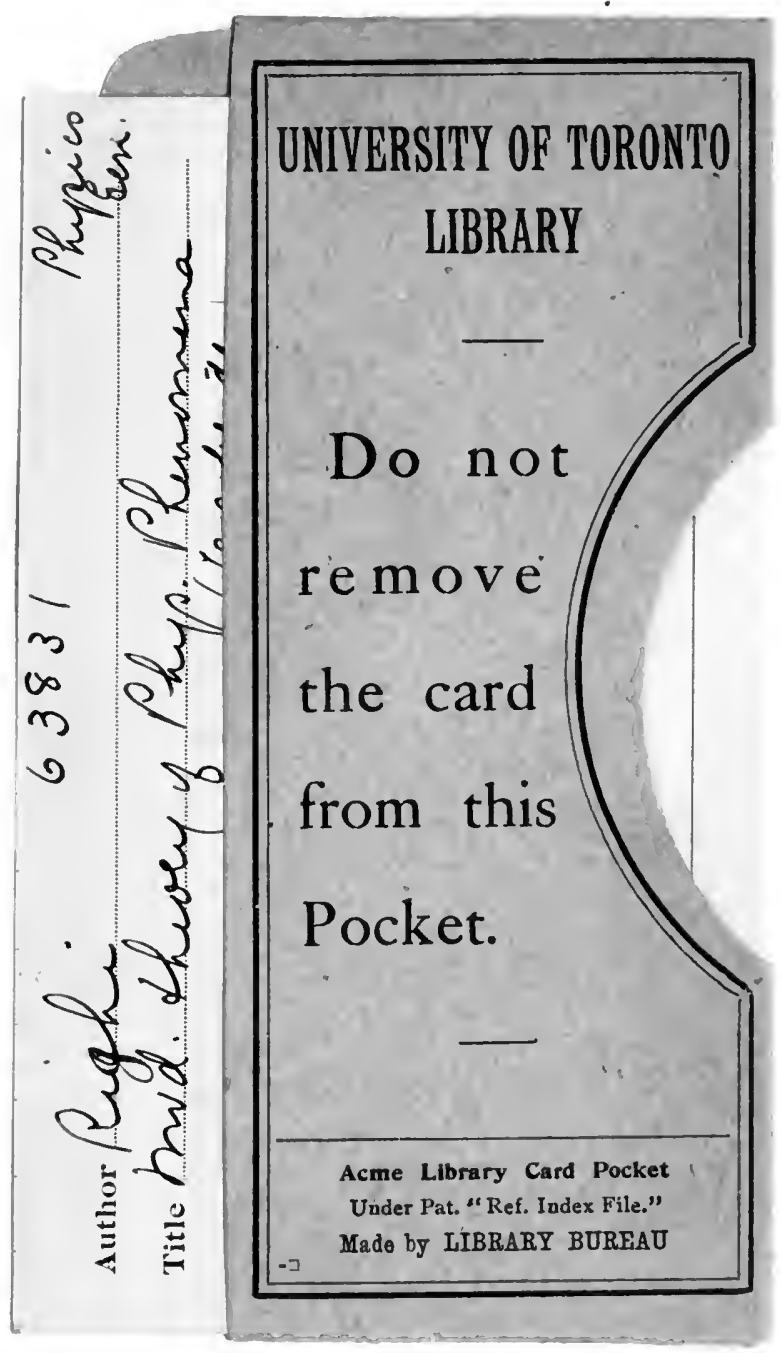


